\title{
Predicting the most deleterious missense nsSNPs of the protein isoforms of the human HLA-G gene and in silico evaluation of their structural and functional consequences
}

\author{
Elaheh Emadi ${ }^{1}$, Fatemeh Akhoundi ${ }^{2}$, Seyed Mehdi Kalantar ${ }^{1}$ and Modjtaba Emadi-Baygi ${ }^{2,3^{*}}$ (D)
}

\begin{abstract}
Background: The Human Leukocyte Antigen G (HLA-G) protein is an immune tolerogenic molecule with 7 isoforms. The change of expression level and some polymorphisms of the HLA-G gene are involved in various pathologies. Therefore, this study aimed to predict the most deleterious missense non-synonymous single nucleotide polymorphisms (nsSNPS) in HLA-G isoforms via in silico analyses and to examine structural and functional effects of the predicted nSSNPS on HLA-G isoforms.

Results: Out of 301 reported SNPs in dbSNP, 35 missense SNPs in isoform 1, 35 missense SNPs in isoform 5, 8 missense SNPs in all membrane-bound HLA-G isoforms and 8 missense SNPs in all soluble HLA-G isoforms were predicted as deleterious by all eight servers (SIFT, PROVEAN, PolyPhen-2, I-Mutant 3.0, SNPs\&GO, PhDSNP, SNAP2, and MUpro). The Structural and functional effects of the predicted nsSNPS on HLA-G isoforms were determined by MutPred2 and HOPE servers, respectively. Consurf analyses showed that the majority of the predicted nsSNPS occur in conserved sites. I-TASSER and Chimera were used for modeling of the predicted nsSNPs. rs 182801644 and rs771111444 were related to creating functional patterns in 5'UTR. 5 SNPs in $3^{\prime} U T R$ of the HLA-G gene were predicted to affect the miRNA target sites. Kaplan-Meier analysis showed the HLA-G deregulation can serve as a prognostic marker for some cancers.

Conclusions: The implementation of in silico SNP prioritization methods provides a great framework for the recognition of functional SNPs. The results obtained from the current study would be called laboratory investigations.
\end{abstract}

Keywords: Deleterious SNPS, HLA-G gene, In silico analysis, Missense mutation, Structural and functional impact

\footnotetext{
* Correspondence: emadi-m@sci.sku.ac.ir

${ }^{2}$ Department of Genetics, Faculty of Basic Sciences, Shahrekord University, Shahrekord, Iran

${ }^{3}$ Research Institute of Biotechnology, Shahrekord University, Shahrekord, Iran

Full list of author information is available at the end of the article
}

C C The Author(s). 2020 Open Access This article is licensed under a Creative Commons Attribution 4.0 International License, which permits use, sharing, adaptation, distribution and reproduction in any medium or format, as long as you give appropriate credit to the original author(s) and the source, provide a link to the Creative Commons licence, and indicate if changes were made. The images or other third party material in this article are included in the article's Creative Commons licence, unless indicated otherwise in a credit line to the material. If material is not included in the article's Creative Commons licence and your intended use is not permitted by statutory regulation or exceeds the permitted use, you will need to obtain permission directly from the copyright holder. To view a copy of this licence, visit http://creativecommons.org/licenses/by/4.0/ The Creative Commons Public Domain Dedication waiver (http://creativecommons.org/publicdomain/zero/1.0/) applies to the data made available in this article, unless otherwise stated in a credit line to the data. 


\section{Background}

Single-Nucleotide Polymorphisms (SNPs) are the most copious type of human genetic sequence alterations that exist throughout the genome [1-3]. A missense mutation is a type of nonsynonymous (nsSNPs) substitution in which the one amino acid is substituted with another and may produce a mutated protein with structural and functional changes that may lead to disease. One of the main challenges for scientists is to identify SNPs that are pathogenic or related to a particular effect in humans [4]. Nowadays, deleterious nsSNPs in the desired gene can be identified using in -silico approaches. These approaches are reliable, user-friendly, fast, and low cost [5].

The major histocompatibility complex (MHC) is a group of genes encoding essential proteins for the adaptive immune system to identify fragments derived from pathogens [6]. In humans, The MHC complex is also called the human leukocyte antigen (HLA) complex [7]. The HLA genes have been classified into 3 classes I, II, and III. MHC class I genes are divided into two groups: major or classical (HLA-A, HLA-B, and HLA-C) and minor or non-classical (HLA-E, HLA-F, and HLA-G) [8], as they differ from each other by their genetic diversity, expression, structure, and functions [9].

The Human Leukocyte Antigen G (HLA-G) is a proteincoding gene on chromosome $6 \mathrm{p} 21.3$ and has an important function in modulation of the immune responses and diseases such as chronic viral infections, autoimmune disorders, transplantation and cancers $[10,11]$.

$H L A-G$ gene displays low polymorphism but several mature mRNAs can be produced as a result of differential splicing of the primary transcript. The mature mRNAs encode 7 different protein isoforms, 4 of them being membrane-bound (HLA-G1 to G4), and 3 soluble or secreted (HLA-G5 to G7) [11]. Also, Roux et al. reported an inventory of novel HLA-G isoforms that have an extended $5^{\prime}$-region and lack the transmembrane and alpha-1 domains [12]. Soluble HLA-G1 (sHLA-G1) protein can be produced through the proteolysis activity of metalloprotease which maintains the functions of the membrane counterpart completely [13]. The general structure of an HLA-G protein consists of a heavy chain of 3 globular domains $(\alpha 1, \alpha 2$, and $\alpha 3)$ and a light chain ( $\beta$-2-microglobulin (B2M)) and a peptide (Fig. 1) [9].

HLA-G is involved in the control of the immune responses to maintain a fetomaternal tolerance in pregnancies [15]. Interaction of immune effector cells with HLA-G often introduces the suppression of them. The effects of inhibition are performed via three ITIMbearing receptors expressed on various immune cells: ILT2/CD85/LIR-1, ILT4/CD85d/LIR-2, and KIR2DL4/ CD158d [9, 16]. ILT2 is expressed by myeloid and lymphoid cells, ILT4 by myeloid cells, and KIR2DL4 by NK and T CD8+ [16]. The binding site of receptors to

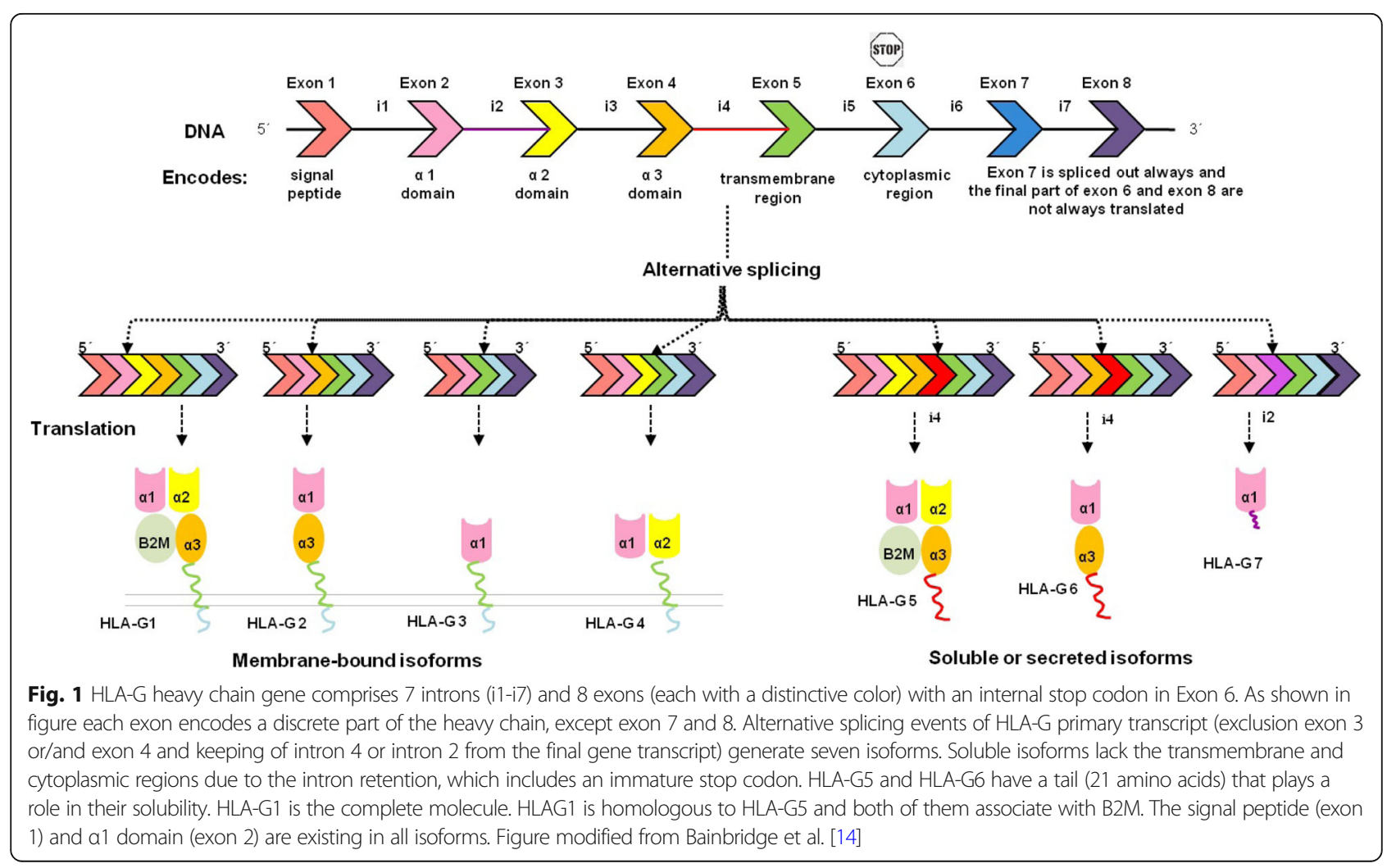


HLA-G is different. Interaction of HLA-G with ILT2 requires the association of the $\alpha 3$ domain with $\beta 2 \mathrm{M}$ but not for binding to ILT4. The KIR2DL4 binds to the $\alpha 1$ domain $[11,15,16]$.

sHLA-G and membrane-bound HLA-G isoforms have alike functions. The membrane-bound HLA-G inhibits peripheral natural killer (NK) cytotoxicity and $\mathrm{CD} 4^{+}$ cells directly through interaction with ILT2. The decidual NK (dNK) cells up-take and internalize HLA-G from the cell membrane of extravillous trophoblast cells through trogocytosis. HLA-G internalization results in maintaining low cytotoxicity and immunosuppressive status of dNK cells to protect the fetus versus dNKs activity and further release of a set of angiogenic factors to promote vascular remodeling and fetal growth at the beginning of pregnancy. The interaction of HLA-G with ILT2s on $\mathrm{CD} 4^{+} \mathrm{T}$ cells decreases the alloproliferative effect of $\mathrm{CD}^{+}{ }^{+} \mathrm{T}$ cells. Binding sHLA-G to $\mathrm{ILT}^{+}{ }^{+} \mathrm{DCs}$ leads to the generation of IL-10 and IL-10-producing DCs can promote the expansion of Tregs $\left(\mathrm{CD} 4^{+}\right.$ CD25 ${ }^{\text {high }}$ FOXP3) and Tr1s differentiation. Besides, the rapid reproduction, differentiation, and antibody production of B cells are inhibited due to HLA-G interplay with the LILRB1s on B cells. Moreover, apoptosis of $\mathrm{CD}^{+} \mathrm{T}$ cells through activation of the FasR/FasL pathway and endothelial cells are induced by HLA-G5 via interactions with $\mathrm{CD} 8$ receptor on $\mathrm{CD}^{+} \mathrm{T}$ cells and $\mathrm{CD} 160$ on endothelial cells [15-17].

$H L A-G$ has a restricted tissue-specific protein expression in normal situations examples being extravillous cytotrophoblasts in the placenta, some immune cells, thymic medulla, and cornea. The neo-expression of HLA-G occurs in different pathological situations [15, 18-20].

The expression of $H L A-G$ gene is adjusted mostly by a unique promoter region in comparison with other $H L A$ genes and also at the post-transcriptional control level [21].

A single nucleotide polymorphism of a gene in the coding region or the regulatory region can lead to disease as a result of the expression change or structural and/or function alteration [4]. Most experimental and pathological studies of the HLA-G gene have been focused on polymorphisms in the promoter and 3' UTR regions. The rate of polymorphisms in the coding sequence of this gene is low that indicates a powerful evolutionary pressure acting on the coding sequence [10].

Polymorphisms in the coding region may change the conformation of protein which could lead to modification of protein function including modulating immune responses, production of isoforms, peptide binding, and ability polymerization. HLA-G expression may change by altering the binding affinity of targeted sequences to transcriptional or post-transcriptional factors considering variations in the HLA-G promoter and $3^{\prime}$ UTR regions [10].
Concerning the important function of HLA-G in health and diseases in human, the main objectives of this study are to predict the most deleterious missense SNPs in HLA-G1 and HLA-G5, the common most deleterious missense SNPs in membrane-bound HLA-G isoforms, the common most deleterious missense SNPs in soluble HLA-G isoforms and finally to evaluate the impacts of the SNPs on the structure and function of HLA-G protein. The current study presents useful information about the most deleterious missense SNPs and their effects on the structure and function of HLA-G protein. In this paper, we also investigated the correlation between the survival rates of patients in some cancer types with HLA-G expression. The various steps of our study are shown in a flow chart (Fig. 2).

\section{Results}

Currently, one of the valuable fields of computational genetic research is the identification of SNPs involved in diseases. At present, the advancement of computational biology methods has enabled us to detect the damaging SNPs in the objective genes. Computational methods are used to study the effect of nsSNPs on protein structure and function at the molecular level [22]. In this study, several computational methods were applied to determine the most deleterious common missense SNPs between soluble HLA-G isoforms and the most deleterious common missense SNPs between membrane-bound HLA-G isoforms as well as the most deleterious missense SNPs in HLA-G1 (the longest isoform protein of the HLA-G gene among membrane-bound HLA-G isoforms) and HLA-G5 isoforms (the longest isoform protein of the HLA-G gene among soluble HLA-G isoforms).

\section{SNP dataset of the HLA-G gene from NCBI dbSNP and protein sequences dataset}

The desired SNPs of the HLA-G gene were retrieved from the NCBI dbSNP database because it is the most extensive SNP database [23]. SNPs retrieved from NCBI and their corresponding IMGT/HLA alleles are shown in the supplementary Table 1 . Of the total reported SNPs in the human HLA-G gene sequence, 301 SNPs are missense (16.38\%), 117 SNPs are in $3^{\prime}$ UTR (6.36\%) and 65 SNPs are in $5^{\prime}$ UTR (3.53\%). A pictorial description of the distribution of SNPs in the HLA-G gene represented in percentage terms is shown in Fig. 3. Most tools for analyzing protein require the amino acid sequence, for this reason, the protein sequences of seven HLA-G isoforms were retrieved from the UniProt database. The seven protein isoforms of HLA-G (HLA-G17) consist of 338, 246, 154, 246, 319, 227, and 116 amino acids respectively, and a 24 -amino acid signal peptide. 


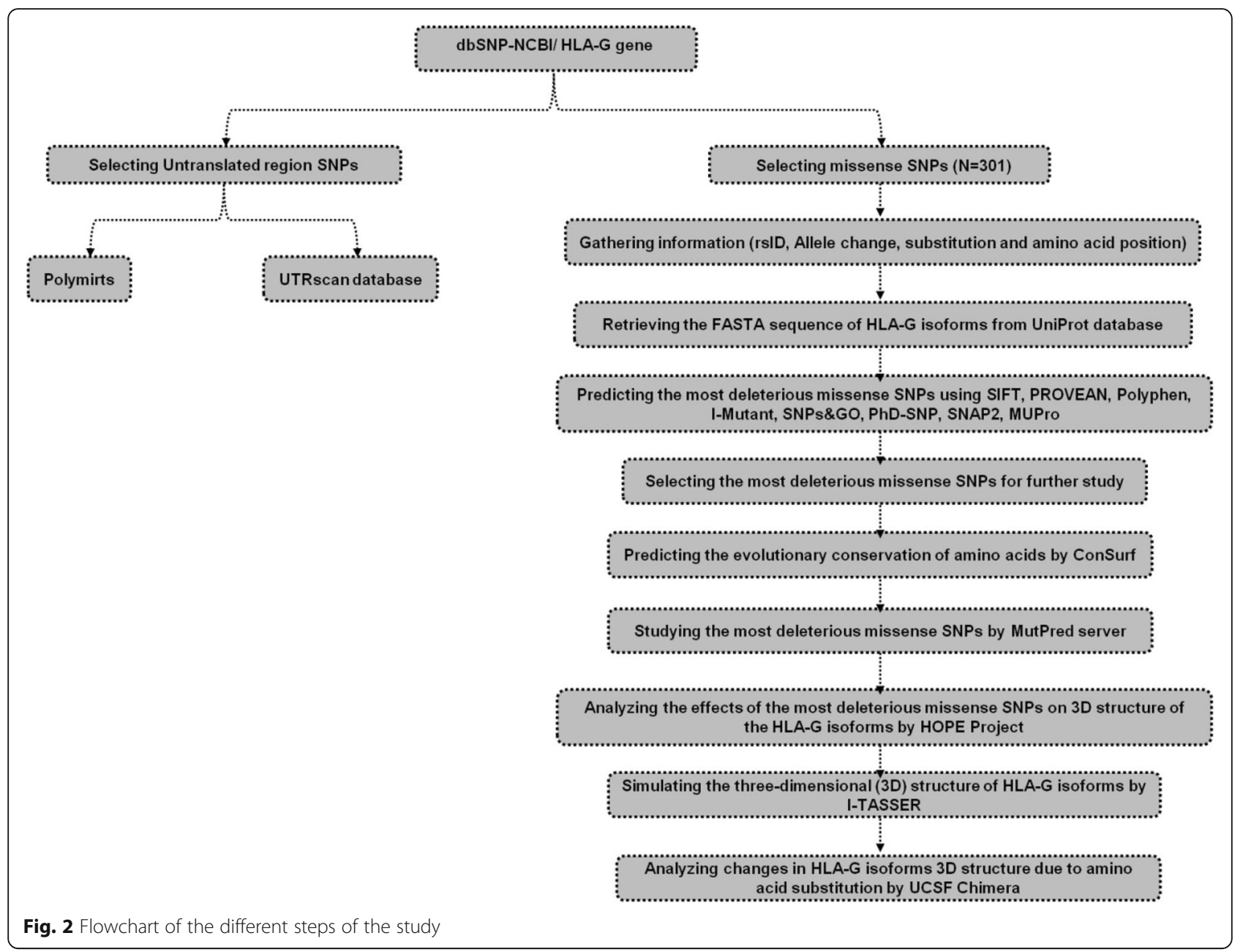

Identification of the most deleterious missense SNPs in HLA-G isoforms using several different servers

At present, there is an extensive range of computational tools used to predict the consequences of missense SNPs on protein structure and function. The in silico methods accuracy for prioritizing candidate deleterious SNPs can

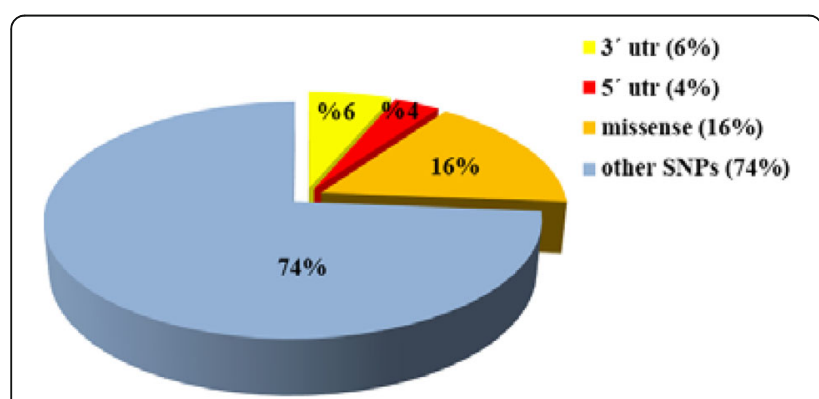

Fig. 3 The 3-D pie-chart shows the percentage of missense SNPs, 5' UTR, 3' UTR and other types of SNPs in HLA-G gene (according to the dbSNP database on December 2018) be enhanced by incorporating the results of diverse computational tools based on various parameters. Hence, we performed the concordance analysis with SIFT, PROVEAN, PolyPhen-2, I-Mutant 3.0, SNPs\&GO, PhD-SNP, SNAP2, and MUpro techniques to predict the most deleterious nsSNPs from the SNP dataset. All the reported missense SNPs for HLA-G were submitted to eight mentioned in silico nsSNP prediction algorithms. We selected missense SNPs that are deleterious in all 8 algorithmic tools manually. Finally, out of total missense SNPs, 35 missense SNPs were predicted as deleterious in isoform 1 (HLA-G1) (Tables 1 and 2), 35 missense SNPs were predicted as deleterious in isoform 5 (HLA-G5) (Supplementary Tables 2 and 3), 8 missense SNPs were predicted as deleterious in all membrane-bound HLA-G isoforms (HLA-G1-4) (Supplementary Tables 4 and 5) and 8 missense SNPs were predicted as deleterious in all soluble HLA-G isoforms (Supplementary Tables 6 and 7) and all further investigations were held for only these missense SNPs. 


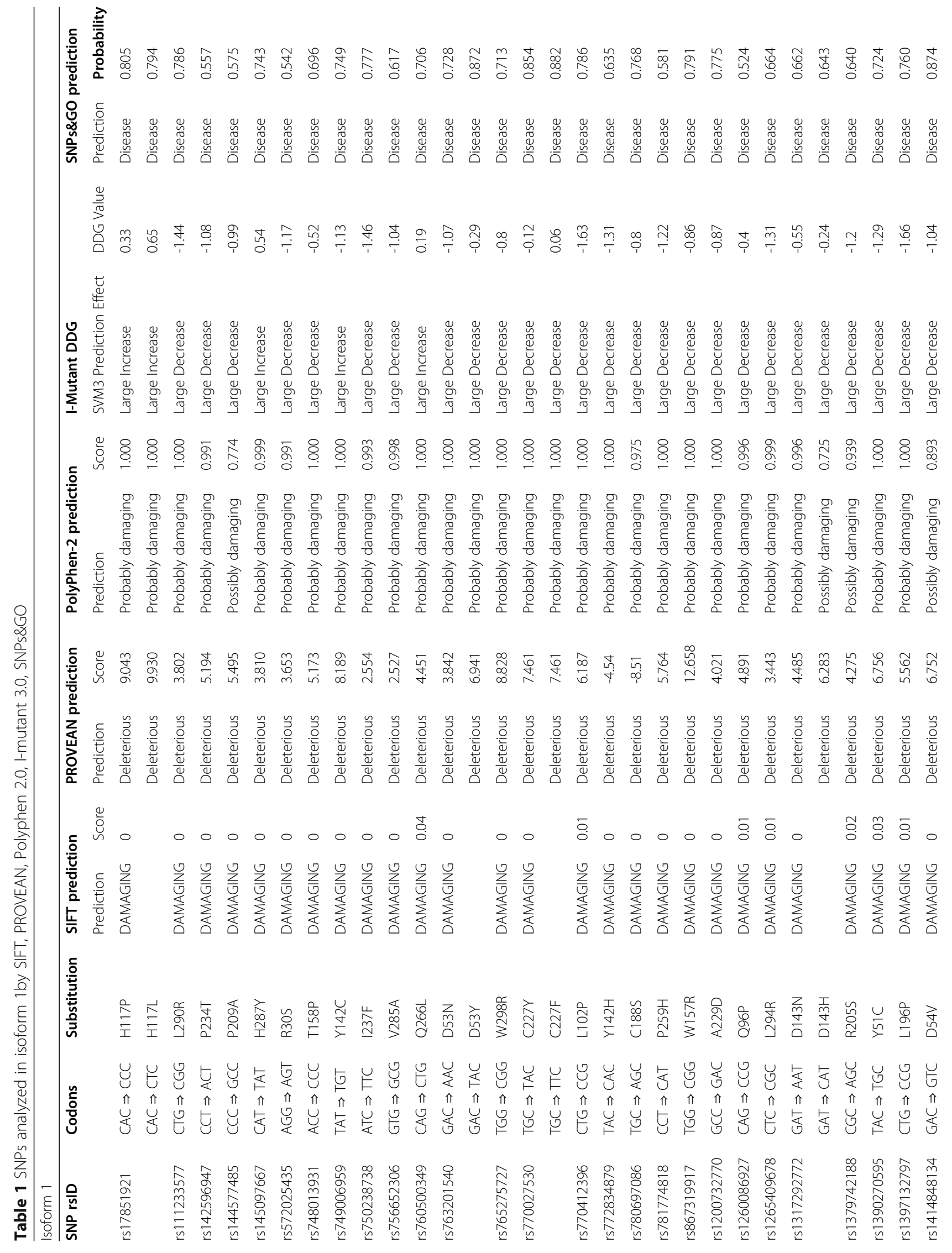




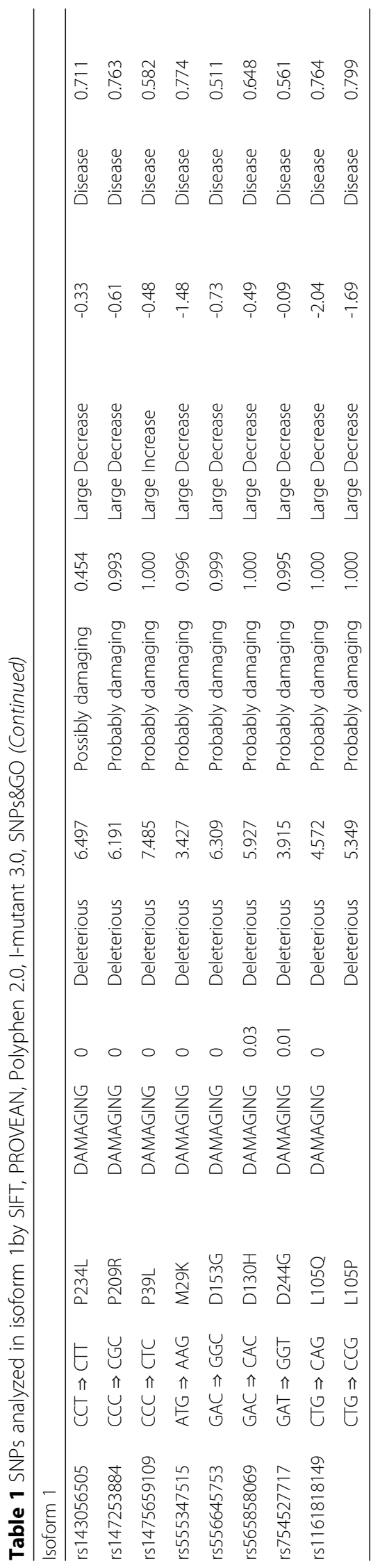


Table 2 SNPs analyzed in isoform 1by PhD-SNP, SNAP2, Mupro

\begin{tabular}{|c|c|c|c|c|c|c|c|c|c|}
\hline \multicolumn{10}{|l|}{ Isoform 1} \\
\hline \multirow[t]{2}{*}{ SNP rsID } & \multirow[t]{2}{*}{ Codons } & \multirow[t]{2}{*}{ Substitution } & \multicolumn{2}{|l|}{ PhD-SNP } & \multicolumn{3}{|l|}{ SNAP2 } & \multicolumn{2}{|l|}{ MUpro } \\
\hline & & & Prediction & Score & Prediction & Score & Expected Accuracy & Prediction & DDG Value \\
\hline \multirow[t]{2}{*}{ rs17851921 } & $C A C \Rightarrow C C C$ & $\mathrm{H} 117 \mathrm{P}$ & Disease & 5 & effect & 94 & $95 \%$ & DECREASE & -0.94483558 \\
\hline & $C A C \Rightarrow C T C$ & $\mathrm{H} 117 \mathrm{~L}$ & Disease & 5 & effect & 82 & $91 \%$ & INCREASE & 0.17248775 \\
\hline rs111233577 & $\mathrm{CTG} \Rightarrow \mathrm{CGG}$ & L290R & Disease & 6 & effect & 52 & $75 \%$ & DECREASE & -1.4553025 \\
\hline rs142596947 & $C C T \Rightarrow A C T$ & P234T & Disease & 8 & effect & 58 & $75 \%$ & DECREASE & -0.8378542 \\
\hline rs144577485 & $\mathrm{CCC} \Rightarrow \mathrm{GCC}$ & P209A & Disease & 4 & effect & 30 & $66 \%$ & DECREASE & -1.3457662 \\
\hline rs145097667 & CAT $\Rightarrow$ TAT & $\mathrm{H} 287 \mathrm{Y}$ & Disease & 6 & effect & 79 & $85 \%$ & INCREASE & 0.00175126 \\
\hline rs572025435 & $A G G \Rightarrow A G T$ & R30S & Disease & 4 & effect & 2 & $53 \%$ & DECREASE & -1.582361 \\
\hline rs748013931 & $\mathrm{ACC} \Rightarrow \mathrm{CCC}$ & T158P & Disease & 3 & effect & 56 & $75 \%$ & DECREASE & -1.0769639 \\
\hline rs749006959 & TAT $\Rightarrow$ TGT & Y142C & Disease & 7 & effect & 69 & $80 \%$ & DECREASE & -1.7925753 \\
\hline rs750238738 & $\mathrm{ATC} \Rightarrow \mathrm{TTC}$ & $1237 \mathrm{~F}$ & Disease & 5 & effect & 45 & $71 \%$ & DECREASE & -0.97093071 \\
\hline rs756652306 & $\mathrm{GTG} \Rightarrow \mathrm{GCG}$ & V285A & Disease & 1 & effect & 36 & $66 \%$ & DECREASE & -0.89978472 \\
\hline rs760500349 & $\mathrm{CAG} \Rightarrow \mathrm{CTG}$ & Q266L & Disease & 6 & effect & 78 & $85 \%$ & DECREASE & -0.05227910 \\
\hline \multirow[t]{2}{*}{ rs763201540 } & $\mathrm{GAC} \Rightarrow \mathrm{AAC}$ & D53N & Disease & 5 & effect & 52 & $75 \%$ & DECREASE & -0.79149414 \\
\hline & $\mathrm{GAC} \Rightarrow \mathrm{TAC}$ & D53Y & Disease & 9 & effect & 84 & $91 \%$ & DECREASE & -0.45294328 \\
\hline rs765275727 & $\mathrm{TGG} \Rightarrow \mathrm{CGG}$ & W298R & Disease & 5 & effect & 87 & $91 \%$ & DECREASE & -1.1870648 \\
\hline \multirow[t]{2}{*}{ rs770027530 } & $\mathrm{TGC} \Rightarrow \mathrm{TAC}$ & C227Y & Disease & 8 & effect & 68 & $80 \%$ & DECREASE & -0.88892871 \\
\hline & $\mathrm{TGC} \Rightarrow \mathrm{TTC}$ & C227F & Disease & 9 & effect & 75 & $85 \%$ & DECREASE & -0.71910267 \\
\hline rs770412396 & $\mathrm{CTG} \Rightarrow \mathrm{CCG}$ & L102P & Disease & 7 & effect & 79 & $85 \%$ & DECREASE & -2.238046 \\
\hline rs772834879 & $\mathrm{TAC} \Rightarrow \mathrm{CAC}$ & $\mathrm{Y} 142 \mathrm{H}$ & Disease & 5 & effect & 72 & $85 \%$ & DECREASE & -2.1531554 \\
\hline rs780697086 & $\mathrm{TGC} \Rightarrow \mathrm{AGC}$ & C188S & Disease & 4 & effect & 75 & $85 \%$ & DECREASE & -1.0332928 \\
\hline rs781774818 & $\mathrm{CCT} \Rightarrow \mathrm{CAT}$ & $\mathrm{P} 259 \mathrm{H}$ & Disease & 5 & effect & 4 & $53 \%$ & DECREASE & -1.1893017 \\
\hline rs867319917 & $\mathrm{TGG} \Rightarrow \mathrm{CGG}$ & W157R & Disease & 3 & effect & 93 & $95 \%$ & DECREASE & -0.91293557 \\
\hline rs1200732770 & $\mathrm{GCC} \Rightarrow \mathrm{GAC}$ & A229D & Disease & 8 & effect & 75 & $85 \%$ & DECREASE & -0.6414612 \\
\hline rs1260086927 & $C A G \Rightarrow C C G$ & Q96P & Disease & 6 & effect & 43 & $71 \%$ & DECREASE & -0.97929591 \\
\hline rs1265409678 & $\mathrm{CTC} \Rightarrow \mathrm{CGC}$ & L294R & Disease & 6 & effect & 57 & $75 \%$ & DECREASE & -1.0427076 \\
\hline \multirow[t]{2}{*}{ rs1317292772 } & GAT $\Rightarrow$ AAT & D143N & Disease & 4 & effect & 22 & $63 \%$ & DECREASE & -1.2006322 \\
\hline & GAT $\Rightarrow$ CAT & D143H & Disease & 9 & effect & 25 & $63 \%$ & DECREASE & -1.2840175 \\
\hline rs1379742188 & $C G C \Rightarrow A G C$ & R205S & Disease & 5 & effect & 34 & $66 \%$ & DECREASE & -0.52183883 \\
\hline rs1390270595 & $\mathrm{TAC} \Rightarrow \mathrm{TGC}$ & Y51C & Disease & 2 & effect & 39 & $66 \%$ & DECREASE & -0.83530596 \\
\hline rs1397132797 & $\mathrm{CTG} \Rightarrow \mathrm{CCG}$ & L196P & Disease & 8 & effect & 58 & $75 \%$ & DECREASE & -2.0384073 \\
\hline rs1414848134 & $\mathrm{GAC} \Rightarrow \mathrm{GTC}$ & D54V & Disease & 5 & effect & 75 & $85 \%$ & DECREASE & -0.37383825 \\
\hline rs1430565057 & $\mathrm{CCT} \Rightarrow \mathrm{CT}$ & P234L & Disease & 7 & effect & 81 & $91 \%$ & INCREASE & 0.3342289 \\
\hline rs1472538844 & $\mathrm{CCC} \Rightarrow \mathrm{CGC}$ & P209R & Disease & 3 & effect & 46 & $71 \%$ & DECREASE & -1.0509858 \\
\hline rs1475659109 & $\mathrm{CCC} \Rightarrow \mathrm{CTC}$ & P39L & Disease & 5 & effect & 29 & $63 \%$ & DECREASE & -0.20370663 \\
\hline rs555347515 & ATG $\Rightarrow$ AAG & M29K & Disease & 6 & effect & 25 & $63 \%$ & DECREASE & -1.7776487 \\
\hline rs556645753 & $\mathrm{GAC} \Rightarrow \mathrm{GGC}$ & D153G & Disease & 2 & effect & 17 & $59 \%$ & DECREASE & -2.384922 \\
\hline rs565858069 & $\mathrm{GAC} \Rightarrow \mathrm{CAC}$ & $\mathrm{D} 130 \mathrm{H}$ & Disease & 5 & effect & 2 & $53 \%$ & DECREASE & -1.0708696 \\
\hline rs754527717 & $\mathrm{GAT} \Rightarrow \mathrm{GGT}$ & D244G & Disease & 2 & effect & 7 & $53 \%$ & DECREASE & -1.7605711 \\
\hline \multirow[t]{2}{*}{ rs1161818149 } & $\mathrm{CTG} \Rightarrow \mathrm{CAG}$ & L105Q & Disease & 3 & effect & 70 & $85 \%$ & DECREASE & -1.8766335 \\
\hline & $\mathrm{CTG} \Rightarrow \mathrm{CCG}$ & L105P & Disease & 6 & effect & 76 & $85 \%$ & DECREASE & -2.1510154 \\
\hline
\end{tabular}




\section{Conservation analysis of the most deleterious nsSNPs in HLA-G isoforms by ConSurf sever}

Evolutionary information is essential to investigate further the possible impacts of deleterious nsSNPs [24]. The ConSurf web server characterizes the evolutionary conservation profile of amino acid residues in the protein and whether each amino acid is exposed (on protein surface) or buried (inside protein core) in the protein structure. For example, our ConSurf analysis showed that D53 is an exposed and conserved residue in all soluble HLA-G isoforms and is predicted to have a functional impact on soluble HLA-G isoforms whereas D53 is a buried and conserved residue in isoform 1 and is predicted a structural residue. The ConSurf server produces a colorimetric conservation score as a result. The residues with the utmost change are shown in blue and the conserved residues are shown in purple. The most highly conserved residues are significant for biological function and changing these residues has functional and structural impacts on the proteins [25]. The ConSurf results are compiled in Tables 3, supplementary Tables 810, Fig. 4, and supplementary Figs. 1-3. The results showed that the majority of the most deleterious nsSNPs (87.5\% in isoform 1 and $86.66 \%$ in isoform 5) occur in conserved sites.
Prediction of structural and functional modifications due to the most deleterious SNPs on the HLA-G isoforms by MutPred server

The SNPs were predicted as most deleterious also investigated by the Mutpard server to predict the functional effects of SNPs. The most deleterious SNPs that were submitted to this server along with their predicted functional and structural effect on isoforms and the resultant probability scores were represented in Table 4 and supplementary Tables 11-13. For example, W157R in HLA-G1 was found to be highly deleterious with a $g$ score of 0.936 and was predicted to cause the alteration in transmembrane protein with a $p$ score of 0.000015 , showing very confident hypothesis. W157R in HLA-G5 was found to be highly deleterious with a $\mathrm{g}$ score of 0.93 and was predicted to induce alteration in ordered interface with a $p$ score of 0.0017 , showing a very confident hypothesis. Gain of sulfation at D53 was predicted at D53Y in all membrane-bound HLA-G isoforms ( $\mathrm{g}=0.746$ and $p=0.0044$ in HLA-G1, $\mathrm{g}=$ 0.628 and $p=0.0088$ in HLA-G2, $\mathrm{g}=0.785$ and $p=0.0051$ in HLA-G3 and $\mathrm{g}=0.75$ and $p=0.0046$ in HLA-G4). Loss of proteolytic cleavage at $\mathrm{R} 30$ was predicted at $\mathrm{M} 29 \mathrm{~K}$ in all soluble HLA-G isoforms $(\mathrm{g}=0.688$ and $p=0.0037$ in HLA-G5, $\mathrm{g}=0.754$ and $p=0.0035$ in HLA-G6 and $\mathrm{g}=$ 0.772 and $p=0.003$ in HLA-G7).

Table 3 Evolutionary conservation pattern of amino acids with solvent accessibility in HLA-G1 by ConSurf server

\begin{tabular}{|c|c|c|c|c|c|c|c|c|c|c|c|}
\hline \\
\hline $\begin{array}{l}\text { Isoform } 1 \\
\text { conservation } \\
\text { score }\end{array}$ & $\begin{array}{l}\text { exposed } \\
\text { or } \\
\text { buried }\end{array}$ & prediction & $\begin{array}{l}\text { conservation } \\
\text { score }\end{array}$ & $\begin{array}{l}\text { exposed } \\
\text { or } \\
\text { buried }\end{array}$ & prediction & $\begin{array}{l}\text { conservation } \\
\text { score }\end{array}$ & $\begin{array}{l}\text { exposed } \\
\text { or } \\
\text { buried }\end{array}$ & prediction & $\begin{array}{l}\text { conservation } \\
\text { score }\end{array}$ & $\begin{array}{l}\text { exposed } \\
\text { or } \\
\text { buried }\end{array}$ & prediction \\
\hline M29 & & & L105 & & & C188 & & & D244 & & \\
\hline 1 (variable) & buried & - & 1 (variable) & buried & - & 9 (conserved) & buried & structural & 7 (conserved) & exposed & - \\
\hline R30 & & & $\mathrm{H} 117$ & & & L196 & & & P259 & & \\
\hline 6 (average) & buried & - & 9 (conserved) & buried & structural & 9 (conserved) & buried & structural & 9 (conserved) & exposed & functional \\
\hline P39 & & & D130 & & & R205 & & & Q266 & & \\
\hline 9 (conserved) & exposed & functional & 8 (conserved) & exposed & functional & 7 (conserved) & exposed & - & 9 (conserved) & exposed & functional \\
\hline Y51 & & & Y142 & & & P209 & & & V285 & & \\
\hline 9 (conserved) & exposed & functional & 9 (conserved) & buried & structural & 9 (conserved) & buried & structural & 9 (conserved) & buried & structural \\
\hline D53 & & & D143 & & & C227 & & & $\mathrm{H} 287$ & & \\
\hline 9 (conserved) & buried & structural & 9 (conserved) & exposed & functional & 9 (conserved) & buried & structural & 9 (conserved) & buried & structural \\
\hline D54 & & & D153 & & & A229 & & & L290 & & \\
\hline 8 (conserved) & exposed & functional & 9 (conserved) & exposed & functional & 9 (conserved) & buried & structural & 9 (conserved) & buried & structural \\
\hline Q96 & & & W157 & & & P234 & & & L294 & & \\
\hline 7 (conserved) & exposed & - & 9 (conserved) & buried & structural & 9 (conserved) & exposed & functional & 6 (average) & buried & - \\
\hline L102 & & & T158 & & & 1237 & & & W298 & & \\
\hline 9 (conserved) & buried & structural & 9 (conserved) & exposed & functional & 9 (conserved) & buried & structural & 8 (conserved) & exposed & functional \\
\hline
\end{tabular}

Conservation score has a range of 1.0 to 9.0. Score 9 represents the most conserved and 1 represents the very variable amino acid. An amino acid, if is preserved and exposed, is a functional residue and if is preserved and buried, is a structural residue 


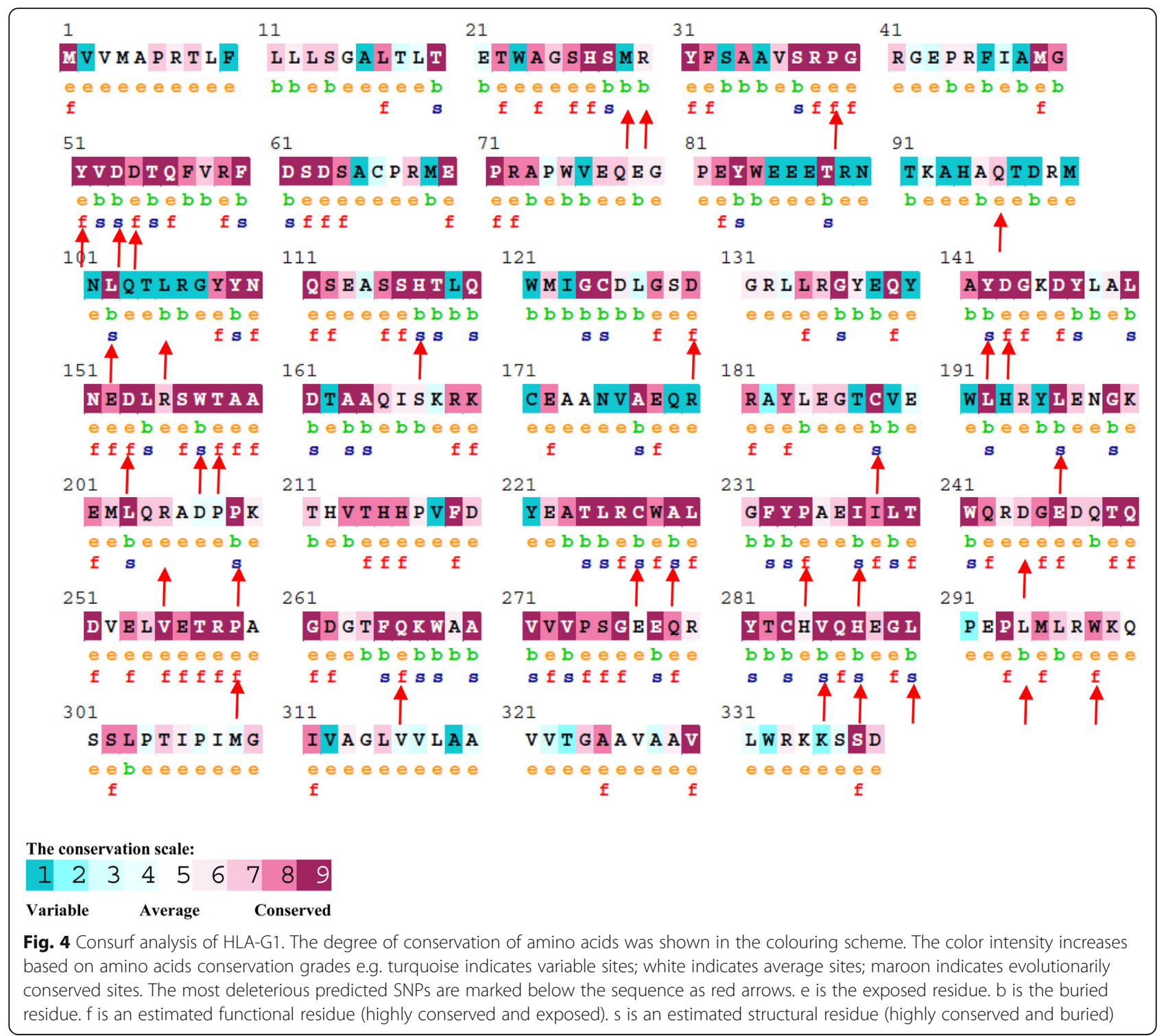

The structural analysis of the most deleterious selected SNPs on HLA-G isoforms by project Hope server

Project HOPE predicted the effects of amino acid substitutions on native structures of HLA-G isoforms, the hydrophobicity, charge, and size change between wildtype and mutant residue and model of the 3D structure. The HOPE reports indicated that there was no exact known structural information for HLA-G1, 3, and 5 isoforms, and HOPE built the models of them based on homologous structures while the 3D-structures of HLAG2, 4, 6 and 7 isoforms were known. All results of the effects of the most deleterious predicted SNPs on structures of the HLA-G isoforms and the difference in physicochemical properties of amino acids of wild type and mutated residue are reported in detail in Additional file 2 and supplementary Tables 14-16. For instance, rs555347515 mutation caused amino acid substitution from methionine into a lysine at the 29th position (M29K). The inspection of this mutation on HLA-G1 showed the mutated residue is bigger than the wild-type residue and probably will not fit in the core of the protein and the mutant residue has a positive charge, while the wild-type residue is neutral, so the positive charge can lead to protein folding problems. Furthermore, the mutation will lead to the loss of hydrophobic interplays in the center of the protein. Additionally, the structural analysis of M29K on HLA-G1 showed this variation is located inside a cluster of residues annotated in UniProt as the Alpha-1 domain and can disturb the domain structure and function (Additional file 2). Moreover, A/ $\mathrm{G}$ mutation (rs556645753) resulted in a change of the aspartic acid to glycine at the 153rd position (D153G). 
Table 4 Prediction of functional effects of the most deleterious SNPS on the HLA-G1 by MutPred

\begin{tabular}{|c|c|c|c|c|c|}
\hline \multicolumn{6}{|c|}{ Isoform 1 (ID: 79bb0a85-d537-4be0-bff8-d0fdb06b5211) } \\
\hline Substitution & $\begin{array}{l}\text { Mutpred score }(>0.50 \text { is } \\
\text { considered pathogenic) }\end{array}$ & Molecular mechanism with $\boldsymbol{p}$-value $<=0.05$ & Probability & $\boldsymbol{P}$-value & prediction \\
\hline \multirow[t]{3}{*}{ H117P } & 0.823 & Altered Metal binding & 0.27 & 0.02 & confident hypotheses \\
\hline & & Altered Transmembrane protein & 0.26 & 1.0e-03 & very confident hypotheses \\
\hline & & Altered Ordered interface & 0.25 & 0.03 & confident hypotheses \\
\hline \multirow[t]{4}{*}{ H117L } & 0.665 & Altered Transmembrane protein & 0.28 & $4.8 \mathrm{e}-04$ & actionable hypotheses \\
\hline & & Loss of Strand & 0.28 & 9.6e-03 & actionable hypotheses \\
\hline & & Altered Metal binding & 0.27 & 0.02 & actionable hypotheses \\
\hline & & Altered Ordered interface & 0.25 & 0.02 & actionable hypotheses \\
\hline L290R & 0.644 & Altered Transmembrane protein & 0.13 & 0.02 & actionable hypotheses \\
\hline \multirow[t]{2}{*}{ P234T } & 0.767 & Altered Ordered interface & 0.32 & $1.8 \mathrm{e}-03$ & very confident hypotheses \\
\hline & & Altered Transmembrane protein & 0.25 & $1.3 e-03$ & very confident hypotheses \\
\hline \multirow[t]{4}{*}{ R30S } & 0.792 & Altered Ordered interface & 0.29 & $5.0 e-03$ & very confident hypotheses \\
\hline & & Loss of Proteolytic cleavage at R30 & 0.21 & $1.2 \mathrm{e}-03$ & very confident hypotheses \\
\hline & & Altered Stability & 0.20 & 0.01 & confident hypotheses \\
\hline & & Altered Metal binding & 0.16 & $3.5 e-03$ & very confident hypotheses \\
\hline \multirow[t]{8}{*}{ T158P } & 0.717 & Altered Ordered interface & 0.32 & $3.6 e-03$ & actionable hypotheses \\
\hline & & Gain of Relative solvent accessibility & 0.29 & 0.01 & actionable hypotheses \\
\hline & & Altered Disordered interface & 0.28 & 0.03 & actionable hypotheses \\
\hline & & Loss of Allosteric site at W157 & 0.28 & $7.4 \mathrm{e}-03$ & actionable hypotheses \\
\hline & & Altered Metal binding & 0.27 & $4.4 \mathrm{e}-03$ & actionable hypotheses \\
\hline & & Altered Transmembrane protein & 0.27 & $6.6 e-04$ & actionable hypotheses \\
\hline & & Loss of Helix & 0.27 & 0.05 & actionable hypotheses \\
\hline & & Altered Coiled coil & 0.10 & 0.04 & actionable hypotheses \\
\hline \multirow[t]{10}{*}{ Y142C } & 0.681 & Altered Metal binding & 0.74 & $1.1 \mathrm{e}-03$ & actionable hypotheses \\
\hline & & Altered Disordered interface & 0.61 & $1.7 e-04$ & actionable hypotheses \\
\hline & & Altered Ordered interface & 0.50 & 1.7e-04 & actionable hypotheses \\
\hline & & Altered Transmembrane protein & 0.29 & 2.1e-04 & actionable hypotheses \\
\hline & & Gain of Relative solvent accessibility & 0.27 & 0.02 & actionable hypotheses \\
\hline & & Loss of Acetylation at K145 & 0.20 & 0.04 & actionable hypotheses \\
\hline & & Loss of Methylation at K145 & 0.17 & 0.01 & actionable hypotheses \\
\hline & & Loss of Ubiquitylation at K145 & 0.16 & 0.04 & actionable hypotheses \\
\hline & & Gain of Disulfide linkage at Y142 & 0.09 & 0.05 & actionable hypotheses \\
\hline & & Loss of Sulfation at Y147 & 0.09 & $3.5 \mathrm{e}-03$ & actionable hypotheses \\
\hline \multirow[t]{5}{*}{$1237 \mathrm{~F}$} & 0.658 & Altered Disordered interface & 0.33 & 0.01 & actionable hypotheses \\
\hline & & Altered Ordered interface & 0.28 & 0.04 & actionable hypotheses \\
\hline & & Altered Transmembrane protein & 0.26 & $1.1 \mathrm{e}-03$ & actionable hypotheses \\
\hline & & Loss of Strand & 0.26 & 0.04 & actionable hypotheses \\
\hline & & Loss of Pyrrolidone carboxylic acid at Q242 & 0.06 & 0.02 & actionable hypotheses \\
\hline \multirow[t]{5}{*}{ Q266L } & 0.444 & Altered Ordered interface & 0.28 & 0.03 & - \\
\hline & & Gain of Relative solvent accessibility & 0.26 & 0.03 & \\
\hline & & Loss of Methylation at K267 & 0.23 & $3.8 \mathrm{e}-03$ & \\
\hline & & Gain of Acetylation at K267 & 0.22 & 0.02 & \\
\hline & & Loss of Pyrrolidone carboxylic acid at Q266 & 0.21 & $1.8 \mathrm{e}-03$ & \\
\hline
\end{tabular}


Table 4 Prediction of functional effects of the most deleterious SNPs on the HLA-G1 by MutPred (Continued)

\begin{tabular}{|c|c|c|c|c|c|}
\hline \multicolumn{6}{|c|}{ Isoform 1 (ID: 79bb0a85-d537-4be0-bff8-d0fdb06b5211) } \\
\hline Substitution & $\begin{array}{l}\text { Mutpred score (>0.50 is } \\
\text { considered pathogenic) }\end{array}$ & Molecular mechanism with $\boldsymbol{p}$-value $<=0.05$ & Probability & $\boldsymbol{P}$-value & prediction \\
\hline & & Altered Metal binding & 0.20 & 0.03 & \\
\hline & & Gain of Catalytic site at D262 & 0.16 & 0.02 & \\
\hline & & Altered Transmembrane protein & 0.15 & 0.01 & \\
\hline & & Gain of Proteolytic cleavage at D262 & 0.14 & 0.02 & \\
\hline \multirow[t]{7}{*}{ D53Y } & 0.746 & Altered Ordered interface & 0.39 & $1.1 \mathrm{e}-03$ & actionable hypotheses \\
\hline & & Altered Disordered interface =) & 0.38 & $6.6 e-03$ & actionable hypotheses \\
\hline & & Altered Metal binding & 0.37 & $1.7 e-03$ & actionable hypotheses actionable \\
\hline & & Altered Transmembrane protein & 0.19 & $6.6 e-03$ & hypotheses \\
\hline & & Loss of Proteolytic cleavage at D53 & 0.12 & 0.03 & actionable hypotheses \\
\hline & & Gain of Pyrrolidone carboxylic acid at Q56 & 0.09 & 0.01 & actionable hypotheses \\
\hline & & Gain of Sulfation at D53 & 0.07 & $4.4 \mathrm{e}-03$ & actionable hypotheses \\
\hline \multirow[t]{2}{*}{ W298R } & 0.822 & Altered Ordered interface & 0.36 & 2.1e-03 & very confident hypotheses \\
\hline & & Altered Transmembrane protein & 0.12 & 0.03 & confident hypotheses \\
\hline \multirow[t]{5}{*}{$\mathrm{C} 227 \mathrm{Y}$} & 0.87 & Altered Disordered interface & 0.42 & $3.2 \mathrm{e}-03$ & very confident hypotheses \\
\hline & & Altered Ordered interface & 0.39 & 4.0e-04 & very confident hypotheses \\
\hline & & Altered Metal binding & 0.34 & $9.6 e-03$ & very confident hypotheses \\
\hline & & Altered Transmembrane protein & 0.31 & $1.2 \mathrm{e}-04$ & very confident hypotheses \\
\hline & & Loss of Helix & 0.28 & 0.03 & confident hypotheses \\
\hline \multirow[t]{5}{*}{$\mathrm{C} 227 \mathrm{~F}$} & 0.888 & Altered Metal binding & 0.37 & $7.8 \mathrm{e}-03$ & very confident hypotheses \\
\hline & & Altered Disordered interface & 0.34 & 0.01 & very confident hypotheses \\
\hline & & Altered Ordered interface & 0.28 & $4.3 e-03$ & very confident hypotheses \\
\hline & & Loss of Helix & 0.27 & 0.04 & confident hypotheses \\
\hline & & Altered Transmembrane protein & 0.26 & $9.6 e-04$ & very confident hypotheses \\
\hline \multirow[t]{7}{*}{ L102P } & 0.735 & Altered Disordered interface & 0.36 & 7.6e-03 & actionable hypotheses \\
\hline & & Gain of Intrinsic disorder & 0.34 & 0.02 & actionable hypotheses \\
\hline & & Altered Transmembrane protein & 0.31 & $1.1 \mathrm{e}-04$ & actionable hypotheses \\
\hline & & Loss of Helix & 0.31 & $4.8 \mathrm{e}-03$ & actionable hypotheses \\
\hline & & Altered DNA binding & 0.25 & $8.9 e-03$ & actionable hypotheses \\
\hline & & Altered Stability & 0.21 & 0.01 & actionable hypotheses \\
\hline & & Loss of Proteolytic cleavage at R99 & 0.13 & 0.02 & actionable hypotheses \\
\hline \multirow[t]{11}{*}{$\mathrm{Y} 142 \mathrm{H}$} & 0.667 & Altered Metal binding & 0.78 & $8.2 \mathrm{e}-04$ & actionable hypotheses \\
\hline & & Altered Ordered interface & 0.44 & $5.4 \mathrm{e}-04$ & actionable hypotheses \\
\hline & & Altered Disordered interface & 0.35 & $8.9 e-03$ & actionable hypotheses \\
\hline & & Altered Transmembrane protein & 0.31 & $1.1 \mathrm{e}-04$ & actionable hypotheses \\
\hline & & Gain of Relative solvent accessibility & 0.25 & 0.03 & actionable hypotheses \\
\hline & & Gain of Acetylation at K145 & 0.20 & 0.04 & actionable hypotheses \\
\hline & & Loss of Methylation at K145 & 0.17 & 0.01 & actionable hypotheses \\
\hline & & Altered Stability & 0.17 & 0.02 & actionable hypotheses \\
\hline & & Loss of Ubiquitylation at K145 ( & 0.15 & 0.05 & actionable hypotheses \\
\hline & & Altered Coiled coil & 0.10 & 0.04 & actionable hypotheses \\
\hline & & Loss of Sulfation at Y147 & 0.09 & $3.5 e-03$ & actionable hypotheses \\
\hline
\end{tabular}


Table 4 Prediction of functional effects of the most deleterious SNPs on the HLA-G1 by MutPred (Continued)

\begin{tabular}{|c|c|c|c|c|c|}
\hline \multicolumn{6}{|c|}{ Isoform 1 (ID: 79bb0a85-d537-4be0-bff8-d0fdb06b5211) } \\
\hline Substitution & $\begin{array}{l}\text { Mutpred score ( }>0.50 \text { is } \\
\text { considered pathogenic) }\end{array}$ & Molecular mechanism with $\boldsymbol{p}$-value $<=0.05$ & Probability & $\boldsymbol{P}$-value & prediction \\
\hline \multirow[t]{4}{*}{ C188S } & \multirow[t]{4}{*}{0.795} & Altered Disordered interface & 0.29 & 0.03 & confident hypotheses \\
\hline & & Altered Ordered interface & 0.24 & 0.03 & confident hypotheses \\
\hline & & Altered DNA binding & 0.17 & 0.03 & confident hypotheses \\
\hline & & Altered Transmembrane protein & 0.09 & 0.05 & confident hypotheses \\
\hline \multirow[t]{6}{*}{$\mathrm{P} 259 \mathrm{H}$} & \multirow[t]{6}{*}{0.607} & Altered Metal binding & 0.47 & $5.9 e-03$ & actionable hypotheses \\
\hline & & Altered Ordered interface & 0.28 & 0.04 & actionable hypotheses \\
\hline & & Loss of Loop & 0.27 & 0.02 & actionable hypotheses \\
\hline & & Altered Transmembrane protein & 0.20 & $5.0 \mathrm{e}-03$ & actionable hypotheses \\
\hline & & Gain of Catalytic site at D262 & 0.13 & 0.03 & actionable hypotheses \\
\hline & & Loss of Proteolytic cleavage at D262 & 0.13 & 0.03 & actionable hypotheses \\
\hline \multirow[t]{7}{*}{ W157R } & \multirow[t]{7}{*}{0.936} & Altered Ordered interface & 0.37 & $1.8 \mathrm{e}-03$ & very confident hypotheses \\
\hline & & Altered Transmembrane protein & 0.36 & $1.5 \mathrm{e}-05$ & very confident hypotheses \\
\hline & & Gain of Relative solvent accessibility & 0.33 & $3.3 e-03$ & very confident hypotheses \\
\hline & & Altered Disordered interface & 0.30 & 0.02 & confident hypotheses \\
\hline & & Loss of Allosteric site at W157 & 0.30 & $3.7 e-03$ & very confident hypotheses \\
\hline & & Altered Metal binding & 0.28 & $3.8 \mathrm{e}-03$ & very confident hypotheses \\
\hline & & Altered Coiled coil & 0.27 & 0.01 & confident hypotheses \\
\hline \multirow[t]{4}{*}{ A229D } & \multirow[t]{4}{*}{0.843} & Altered Transmembrane protein & 0.34 & $2.4 \mathrm{e}-05$ & very confident hypotheses \\
\hline & & Altered Metal binding & 0.29 & 0.02 & confident hypotheses \\
\hline & & Gain of Relative solvent accessibility & 0.28 & 0.02 & confident hypotheses \\
\hline & & Altered Ordered interface & 0.27 & $9.8 \mathrm{e}-03$ & very confident hypotheses \\
\hline \multirow[t]{6}{*}{ Q96P } & \multirow[t]{6}{*}{0.683} & Loss of Helix & 0.30 & $9.0 \mathrm{e}-03$ & actionable hypotheses \\
\hline & & Altered Transmembrane protein & 0.29 & $3.0 \mathrm{e}-04$ & actionable hypotheses \\
\hline & & Altered DNA binding & 0.25 & $6.7 e-03$ & actionable hypotheses \\
\hline & & Gain of Ubiquitylation at K92 & 0.15 & 0.04 & actionable hypotheses \\
\hline & & Gain of Proteolytic cleavage at R99 & 0.13 & 0.02 & actionable hypotheses \\
\hline & & Loss of Pyrrolidone carboxylic acid at Q96 & 0.10 & 0.01 & actionable hypotheses \\
\hline L294R & 0.527 & Altered Ordered interface & 0.27 & $8.6 \mathrm{e}-03$ & actionable hypotheses \\
\hline \multirow[t]{9}{*}{ D143N } & \multirow[t]{9}{*}{0.649} & Altered Metal binding & 0.38 & $1.5 \mathrm{e}-03$ & actionable hypotheses \\
\hline & & Altered Transmembrane protein & 0.28 & $3.8 \mathrm{e}-04$ & actionable hypotheses \\
\hline & & Altered Ordered interface & 0.28 & 0.04 & actionable hypotheses \\
\hline & & Altered Disordered interface & 0.28 & 0.03 & actionable hypotheses \\
\hline & & Gain of Relative solvent accessibility & 0.26 & 0.03 & actionable hypotheses \\
\hline & & Loss of Acetylation at K145 & 0.19 & 0.05 & actionable hypotheses \\
\hline & & Loss of Methylation at K145 & 0.16 & 0.01 & actionable hypotheses \\
\hline & & Loss of Ubiquitylation at K145 & 0.15 & 0.04 & actionable hypotheses \\
\hline & & Loss of Sulfation at Y147 & 0.09 & $3.4 \mathrm{e}-03$ & actionable hypotheses \\
\hline \multirow[t]{5}{*}{ D143H } & \multirow[t]{5}{*}{0.738} & Altered Metal binding & 0.43 & $7.0 \mathrm{e}-03$ & actionable hypotheses \\
\hline & & Altered Transmembrane protein & 0.32 & $7.8 \mathrm{e}-05$ & actionable hypotheses \\
\hline & & Altered Disordered interface & 0.29 & 0.03 & actionable hypotheses \\
\hline & & Altered Ordered interface & 0.27 & 7.0e-03 & actionable hypotheses \\
\hline & & Loss of Relative solvent accessibility & 0.26 & 0.03 & actionable hypotheses \\
\hline
\end{tabular}


Table 4 Prediction of functional effects of the most deleterious SNPs on the HLA-G1 by MutPred (Continued)

\begin{tabular}{|c|c|c|c|c|c|}
\hline Substitution & $\begin{array}{l}\text { Mutpred score }(>0.50 \text { is } \\
\text { considered pathogenic) }\end{array}$ & Molecular mechanism with $\boldsymbol{p}$-value $<=0.05$ & Probability & $\boldsymbol{P}$-value & prediction \\
\hline & & Loss of Acetylation at K145 & 0.19 & 0.05 & actionable hypotheses \\
\hline & & Loss of Methylation at K145 & 0.17 & 0.01 & actionable hypotheses \\
\hline & & Loss of Ubiquitylation at K145 & 0.16 & 0.03 & actionable hypotheses \\
\hline & & Loss of Sulfation at Y147 & 0.09 & 3.3e-03 & actionable hypotheses \\
\hline \multirow[t]{3}{*}{ R205S } & 0.617 & Gain of Intrinsic disorder & 0.34 & 0.02 & actionable hypotheses \\
\hline & & Altered Ordered interface & 0.29 & 0.03 & actionable hypotheses \\
\hline & & Loss of Helix & 0.27 & 0.05 & actionable hypotheses \\
\hline \multirow[t]{9}{*}{ Y51C } & 0.715 & Altered Disordered interface & 0.60 & $1.9 \mathrm{e}-04$ & actionable hypotheses \\
\hline & & Altered Metal binding & 0.53 & 4.1e-03 & actionable hypotheses \\
\hline & & Altered Ordered interface & 0.30 & 4.6e-03 & actionable hypotheses \\
\hline & & Loss of Strand & 0.27 & 0.02 & actionable hypotheses \\
\hline & & Altered Transmembrane protein & 0.20 & $5.0 \mathrm{e}-03$ & actionable hypotheses \\
\hline & & Loss of Proteolytic cleavage at D53 & 0.12 & 0.03 & actionable hypotheses \\
\hline & & Altered Stability & 0.12 & 0.03 & actionable hypotheses \\
\hline & & Gain of Pyrrolidone carboxylic acid at Q56 & 0.08 & 0.02 & actionable hypotheses \\
\hline & & Loss of Sulfation at Y51 & 0.03 & 0.02 & actionable hypotheses \\
\hline \multirow[t]{4}{*}{ L196P } & 0.884 & Altered Disordered interface & 0.34 & 0.01 & confident hypotheses \\
\hline & & Altered Ordered interface & 0.27 & $9.0 \mathrm{e}-03$ & very confident hypotheses \\
\hline & & Altered DNA binding & 0.16 & 0.04 & confident hypotheses \\
\hline & & Altered Stability & 0.14 & 0.02 & confident hypotheses \\
\hline \multirow[t]{7}{*}{ D54V } & 0.718 & Altered Metal binding & 0.55 & $3.1 \mathrm{e}-04$ & actionable hypotheses \\
\hline & & Altered Disordered interface & 0.40 & 4.2e-03 & actionable hypotheses \\
\hline & & Altered Ordered interface & 0.25 & 0.02 & actionable hypotheses \\
\hline & & Altered Transmembrane protein & 0.14 & 0.02 & actionable hypotheses \\
\hline & & Loss of Proteolytic cleavage at D53 & 0.12 & 0.03 & actionable hypotheses \\
\hline & & Gain of Pyrrolidone carboxylic acid at Q56 & 0.09 & 0.01 & actionable hypotheses \\
\hline & & Loss of Sulfation at Y51 & 0.03 & 0.02 & actionable hypotheses \\
\hline \multirow[t]{4}{*}{ P234L } & 0.809 & Altered Ordered interface & 0.33 & $1.5 \mathrm{e}-03$ & very confident hypotheses \\
\hline & & Altered Disordered interface & 0.30 & 0.03 & confident hypotheses \\
\hline & & Altered Transmembrane protein & 0.29 & $2.0 \mathrm{e}-04$ & very confident hypotheses \\
\hline & & Loss of Strand & 0.28 & 0.01 & confident hypotheses \\
\hline \multirow[t]{5}{*}{ P39L } & 0.659 & Altered Disordered interface & 0.27 & 0.04 & actionable hypotheses \\
\hline & & Loss of B-factor & 0.26 & 0.04 & actionable hypotheses \\
\hline & & Altered Transmembrane protein & 0.24 & $1.6 \mathrm{e}-03$ & actionable hypotheses \\
\hline & & Altered DNA binding & 0.23 & $9.6 e-03$ & actionable hypotheses \\
\hline & & Gain of Proteolytic cleavage at R38 & 0.14 & 0.02 & actionable hypotheses \\
\hline \multirow[t]{5}{*}{ M29K } & 0.720 & Altered Ordered interface & 0.26 & 0.02 & actionable hypotheses \\
\hline & & Loss of Proteolytic cleavage at R30 & 0.20 & $2.6 \mathrm{e}-03$ & actionable hypotheses \\
\hline & & Altered Stability & 0.13 & 0.03 & actionable hypotheses \\
\hline & & Altered Transmembrane protein & 0.12 & 0.03 & actionable hypotheses \\
\hline & & Altered Metal binding & 0.11 & $7.9 \mathrm{e}-03$ & actionable hypotheses \\
\hline
\end{tabular}


Table 4 Prediction of functional effects of the most deleterious SNPs on the HLA-G1 by MutPred (Continued)

\begin{tabular}{|c|c|c|c|c|c|}
\hline \multicolumn{6}{|c|}{ Isoform 1 (ID: 79bb0a85-d537-4be0-bff8-d0fdb06b5211) } \\
\hline Substitution & $\begin{array}{l}\text { Mutpred score }(>0.50 \text { is } \\
\text { considered pathogenic) }\end{array}$ & Molecular mechanism with $\boldsymbol{p}$-value $<=0.05$ & Probability & $\boldsymbol{P}$-value & prediction \\
\hline \multirow[t]{9}{*}{ D153G } & \multirow[t]{9}{*}{0.853} & Altered Metal binding & 0.45 & $1.6 \mathrm{e}-04$ & very confident hypotheses \\
\hline & & Altered Disordered interface & 0.35 & $9.1 \mathrm{e}-03$ & very confident hypotheses \\
\hline & & Loss of Relative solvent accessibility & 0.30 & $8.2 \mathrm{e}-03$ & very confident hypotheses \\
\hline & & Gain of Strand & 0.30 & $3.1 \mathrm{e}-03$ & very confident hypotheses \\
\hline & & Altered Transmembrane protein & 0.28 & $4.6 \mathrm{e}-04$ & very confident hypotheses \\
\hline & & Altered Ordered interface & 0.26 & 0.01 & confident hypotheses \\
\hline & & Gain of Allosteric site at W157 & 0.26 & 7.0e-03 & very confident hypotheses \\
\hline & & Altered Stability & 0.19 & 0.01 & confident hypotheses \\
\hline & & Altered Coiled coil & 0.10 & 0.04 & confident hypotheses \\
\hline \multirow[t]{7}{*}{$\mathrm{D} 130 \mathrm{H}$} & \multirow[t]{7}{*}{0.724} & Altered Transmembrane protein & 0.30 & $1.9 \mathrm{e}-04$ & actionable hypotheses \\
\hline & & Altered Disordered interface & 0.28 & 0.03 & actionable hypotheses \\
\hline & & Altered Metal binding & 0.25 & 0.03 & actionable hypotheses \\
\hline & & Loss of Relative solvent accessibility & 0.25 & 0.04 & actionable hypotheses \\
\hline & & Altered Ordered interface & 0.24 & 0.04 & actionable hypotheses \\
\hline & & Gain of Disulfide linkage at C125 & 0.10 & 0.05 & actionable hypotheses \\
\hline & & Gain of Catalytic site at D126 & 0.08 & 0.05 & actionable hypotheses \\
\hline \multirow[t]{8}{*}{ D244G } & \multirow[t]{8}{*}{0.705} & Altered Ordered interface & 0.30 & 0.02 & actionable hypotheses \\
\hline & & Altered Disordered interface & 0.28 & 0.04 & actionable hypotheses \\
\hline & & Gain of Strand & 0.27 & 0.02 & actionable hypotheses \\
\hline & & Altered Transmembrane protein & 0.26 & $8.2 \mathrm{e}-04$ & actionable hypotheses \\
\hline & & Altered Metal binding & 0.18 & 0.03 & actionable hypotheses \\
\hline & & Gain of Pyrrolidone carboxylic acid at Q248 & 0.15 & $4.6 \mathrm{e}-03$ & actionable hypotheses \\
\hline & & Loss of Proteolytic cleavage at R243 & 0.12 & 0.03 & actionable hypotheses \\
\hline & & Loss of Catalytic site at R243 & 0.09 & 0.04 & actionable hypotheses \\
\hline \multirow[t]{7}{*}{ L105Q } & \multirow[t]{7}{*}{0.507} & Altered Disordered interface & 0.32 & 0.01 & actionable hypotheses \\
\hline & & Gain of Intrinsic disorder & 0.30 & 0.05 & actionable hypotheses \\
\hline & & Altered Transmembrane protein & 0.29 & $2.1 \mathrm{e}-04$ & actionable hypotheses \\
\hline & & Altered Ordered interface & 0.26 & 0.01 & actionable hypotheses \\
\hline & & Altered DNA binding & 0.16 & 0.04 & actionable hypotheses \\
\hline & & Altered Stability & 0.15 & 0.02 & actionable hypotheses \\
\hline & & Loss of N-linked glycosylation at N110 & 0.05 & 0.02 & actionable hypotheses \\
\hline \multirow[t]{7}{*}{ L105P } & \multirow[t]{7}{*}{0.702} & Altered Disordered interface & 0.33 & 0.01 & actionable hypotheses \\
\hline & & Loss of Helix & 0.29 & 0.01 & actionable hypotheses \\
\hline & & Altered Transmembrane protein & 0.28 & $3.7 e-04$ & actionable hypotheses \\
\hline & & Altered Ordered interface & 0.26 & 0.01 & actionable hypotheses \\
\hline & & Altered Stability & 0.20 & 0.01 & actionable hypotheses \\
\hline & & Altered DNA binding & 0.16 & 0.04 & actionable hypotheses \\
\hline & & Gain of N-linked glycosylation at N110 & 0.05 & 0.02 & actionable hypotheses \\
\hline
\end{tabular}


The inspection of this mutation on HLA-G5 showed the mutated residue is smaller than the wild-type residue and this might induce loss of interplays and a further hydrophobic residue that can lead to loss of hydrogen bonds and disturb correct confirmation. The negative charge of the wild-type residue will be lost upon this mutation and this can lead to loss of interactions with other molecules or residues. Moreover, the structural analysis of D153G on HLA-G5 showed this variation is located inside a cluster of residues annotated in UniProt as the Alpha-2 domain and can distract this domain and disturb its function. Glycines are very flexible and can abolish the needed rigidity of HLA-G5 in this area (supplementary Table 14).

\section{Modeling of protein}

I-TASSER tool created the 5 high-quality 3D structures for each HLA-G isoform from its amino acid sequence. We submitted the protein sequence of each isoform without signal peptide as an input to I-TASSER because there were no most deleterious SNPs in the peptide signal sequence and removing signal peptide from the protein sequence can improve the speed of I-TASSER simulation without loss of modeling accuracy. I-TASSER used the top 10 templates which are structurally closest to query protein sequence to model the protein (supplementary Table 17). Among the 5 predicted models for each HLA-G isoform, the first model was selected because it had the highest confidence score (C-score) and it was used for further investigation using Chimera (Additional file 3). A greater level of C-score indicates a model with great confidence and conversely.

\section{Chimera software}

Chimera viewer was utilized to visualize the structures of the HLA-G isoforms using the first model as predicted by I-TASSER (Additional file 4). Furthermore, the structural characteristics of amino acids in wild and mutant protein chains were visualized by Chimera (Additional file 5 and supplementary Tables 18-20). A physicochemical rationale may be presented for the impact on protein activity by visualizing the location of the mutant amino acids [26].

\section{Functional SNPs in UTR predicted by UTRscan tool}

The total of the UTR SNPs was investigated by applying UTRscan. Then analyzing the functional elements for every UTR SNP, the result showed that rs182801644 was related to the creation of functional pattern of uORF, and rs771111444 was related to the creation of a functional patterns of uORF and IRES in 5'UTR (Table 5). The internal ribosome entry site (IRES) is an alternative translation initiation mechanism in a capindependent process in comparison with the ordinary 5 '-cap dependent ribosome scanning mechanism [27]. Upstream open reading frames (uORF) is in the 5'UTR of mRNA that can regulate eukaryotic gene expression [28].

\section{The functional SNPs located in 3'UTRs region predicted by PolymiTRS}

3' untranslated regions (UTR) as the putative target site for miRNAs is a significant gene expression regulator. The SNP in the 3' UTR region may disrupt and/or create miRNA target sites. PolymiRTS database predicted functional SNPs in 3' UTR of the HLA-G gene. Among all the SNPs in the 3'UTR region of the HLAG gene, 5 functional SNPs were predicted to affect the miRNA target sites. The details of the effect of these SNPs on the miRNA sites are listed in Table 6. Two SNPs, rs17179101 and rs1063320 disrupt 9 miRNA conserved sites (ancestral allele with support $\geq 2$ ), while all of them produce 15 novel miRNA target sites.

\section{Protein-protein interactions analysis}

The mutation may change the structure of a protein and thus the function of protein may change. Therefore, mutated protein may interact with other proteins and lead to phenotypic effects. To investigate the interaction of HLA$G$ with various proteins, the STRING server was used. The interaction analysis revealed that HLAG is related to Beta-2-microglobulin (B2M), Leukocyte immunoglobulinlike receptor subfamily B member 2 (LILRB2), Leukocyte immunoglobulin-like receptor subfamily $B$ member 1 (LILRB1), Killer cell immunoglobulin-like receptor 2DL4 (KIR2DL4), HLA class I histocompatibility antigen, alpha chain F (HLA-F), HLA class I histocompatibility antigen, A-3 alpha chain (HLA-A), HLA class I histocompatibility antigen, $\mathrm{Cw}-7$ alpha chain (HLA-C), HLA class I histocompatibility antigen, alpha chain E(HLA-E), HLA class I histocompatibility antigen, B-7 alpha chain (HLA-B), Tcell surface glycoprotein CD8 alpha chain (CD8A) (Fig. 5).

Table 5 Table of HLA-G UTR SNPs with functional importance that were predicted by UTRscan tool

\begin{tabular}{llll}
\hline SNP ID & Nucleotide change & UTR position & Functional element change \\
\hline rs182801644 & C/T & $5^{\prime}$ UTR & no pattern $\rightarrow$ uORF \\
rs771111444 & C/G & $5^{\prime}$ UTR & no pattern $\rightarrow$ IRES \\
& & & no pattern $\rightarrow$ uORF \\
\hline
\end{tabular}


Table 6 Prediction results of PolymiRTS database

\begin{tabular}{|c|c|c|c|c|}
\hline$\overline{d b S N P I D}$ & $\mathrm{miR} I \mathrm{D}$ & Conservation & miRSite & FunctionClass \\
\hline \multirow[t]{2}{*}{ rs1707 } & hsa-miR-5702 & 2 & CTGACTCctcttt & C \\
\hline & hsa-miR-583 & 3 & ctgactCCTCTाT & C \\
\hline \multirow[t]{15}{*}{ rs17179101 } & hsa-miR-4417 & 2 & AGCCCACccctgt & D \\
\hline & hsa-miR-4651 & 5 & agcCCACCCCtgt & D \\
\hline & hsa-miR-541-3p & 2 & aGCCCACCcctgt & D \\
\hline & hsa-miR-608 & 2 & $\operatorname{agcCCACCCCtgt}$ & D \\
\hline & hsa-miR-654-5p & 2 & aGCCCACCcctgt & D \\
\hline & hsa-miR-6756-5p & 2 & agCCCACCCctgt & D \\
\hline & hsa-miR-6766-5p & 2 & agCCCACCCctgt & D \\
\hline & hsa-miR-6782-5p & 3 & agccCACCCCTgt & D \\
\hline & hsa-miR-1587 & 2 & AGCCCAAccctgt & C \\
\hline & hsa-miR-296-3p & 6 & agccCAACCCTgt & C \\
\hline & hsa-miR-3147 & 2 & aGCCCAACcctgt & C \\
\hline & hsa-miR-3620-5p & 2 & AGCCCAAccctgt & C \\
\hline & hsa-miR-4674 & 2 & AGCCCAAccctgt & C \\
\hline & hsa-miR-6823-5p & 3 & agcccAACCCTGt & C \\
\hline & hsa-miR-92a-1-5p & 2 & agCCCAACCctgt & C \\
\hline rs180827037 & hsa-miR-875-3p & 5 & $\operatorname{tttcct} \mathbf{T} T \mathrm{TCCAG}$ & C \\
\hline \multirow[t]{2}{*}{ rs138249160 } & hsa-miR-25-5p & 2 & tCTCCGCCtctgt & C \\
\hline & hsa-miR-6087 & 3 & tctCCGCCTCtgt & C \\
\hline \multirow[t]{4}{*}{ rs1063320 } & hsa-miR-3619-3p & 3 & $\operatorname{tgTGGTCCActga~}$ & C \\
\hline & hsa-miR-4776-5p & 3 & $\operatorname{tgTGGTCCActga~}$ & C \\
\hline & hsa-miR-4800-5p & 3 & $\operatorname{tgtGGTCCACtga}$ & C \\
\hline & hsa-miR-767-5p & 3 & $\operatorname{tgTGGTGCActga~}$ & $D$ \\
\hline
\end{tabular}

In miRsite, sequences of the miRNA sites were shown. The capital letters show bases complementary to the seed region and SNPs were shown in bold font.

The effect of high and low expression levels of HLA-G on overall survival (OS) in patients with various cancers

Kaplan-Meier plotter was exerted to analyze the prognostic value of the HLA-G gene expression for breast, ovarian, lung, and gastric cancers by combining gene expression and cancer patient survival. The subjects were divided into 2 categories (high or low expression levels) according to the median expression of HLA-G. Subsequently, the correlation of expression levels and cancer patient's overall survival rate was evaluated using the Kaplan-Meier plotter. Hazard ratio (HR) with 95\% confidence intervals $(\mathrm{CI})$ and logrank $p$-value were calculated.

HLA-G gene in breast cancer had a hazard ratio $(\mathrm{HR})=0.85(95 \% \mathrm{CI}, 0.69-1.06)$ and logrank $p$-value $=$ 0.15 ; therefore the result was not statistically significant (HLA-G deregulation had not the prognostic value). HLA-G gene in ovarian cancer had an $\mathrm{HR}=0.81(95 \%$ $\mathrm{CI}, 0.71-0.93$ ) and logrank $\mathrm{p}$-value $=0.0023$; therefore the result was statistically significant (the relation between the high expression of HLA-G gene and more survival rate). HLA-G gene in lung cancer had a $H R=$
1.21 (95\% CI, 1.07-1.38) and logrank p-value $=0.0029$ and in gastric cancer $\mathrm{HR}=1.3$ (95\% CI, 1.09-1.54) and logrank $\mathrm{p}$-value $=0.0027$; therefore the results were statistically significant (the relation between the low expression of HLA-G gene and more survival rate) (Fig. 6). The results showed that HLA-G deregulation has distinct implications in different types of cancers. This study shows, the HLA-G deregulation can serve as a prognostic marker for patients with ovarian, lung, and gastric cancer but not for breast cancer.

\section{Discussion}

A large number of SNPs have been distributed throughout the human genome. Increasing evidence has suggested that SNPs are important and valuable in the search for the etiologies of human diseases/traits, the drug design, and human drug response [29, 30]. But the large number of SNPs causes a challenge for scientists because studying all SNPs with molecular approaches to choose target SNPs is an expensive, time-consuming and laborious task [29, 31, 32]. A better sense of genetic 


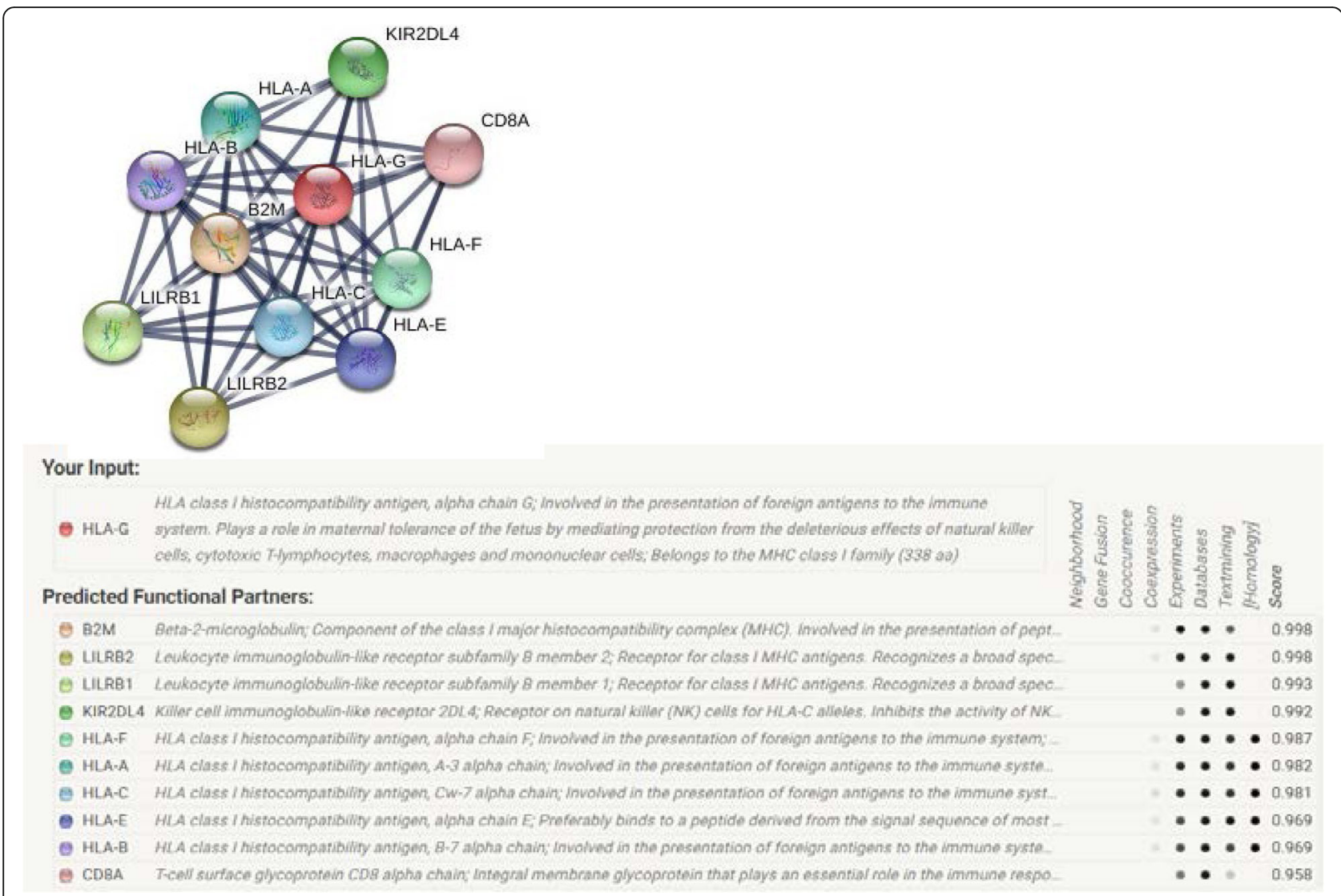

Fig. 5 Protein-protein interaction network of HLAG with 10 partners

variations in susceptibility to disease and their phenotypic effects and reducing the number of them that should be screened in molecular studies may be provided by applying in silico methods [26, 33]. Among SNPs, missense SNPs are correlated with single amino acid substitution in the coded protein as a result of single nucleotide change in a codon that may have an intense impact on the structure and functionality of the relevant protein [4]. There is considerable data about SNPs in the dbSNP/NCBI database [34]. There were 301 missense mutations in the coding region of human HLA-G gene and in this study we focused on them in order to identify the most deleterious missense mutations that could modify the structure and function of the HLA-G isoforms. Identification of functional missense mutations and their role(s) may allow an individualized method for therapeutic goals [10]. HLA-G acts as an immune tolerogenic molecule, playing a role in various pathologies [10]. HLA-G primary mRNA is spliced into seven alternative mRNAs that encode 7 different isoforms of HLA-G protein: four membrane-bound (HLAG1 to G4) and three soluble (HLA-G5 to G7) protein isoforms [35]. Full-length HLA-G protein exhibits a heavy chain consisting of $\alpha 1$ (residues 25 to 114), $\alpha 2$ (residues 115 to 206) and $\alpha 3$ (residues 207 to 298) domains and a light chain (B2M) $[15,36]$.

The HLA-Gl isoform consists of $\alpha 1, \alpha 2$ and $\alpha 3$ domains, transmembrane and cytoplasmic regions. The HLA-G2 isoform lacks the $\alpha 2$ domain. The HLA-G3 isoform does not comprise both the $\alpha 2$ and $\alpha 3$ domains. The HLA-G4 isoform lacks the $\alpha 3$ domain. The HLAG5 isoform comprises the $\alpha 1, \alpha 2$ and $\alpha 3$ domains and lacks transmembrane and cytoplasmic domains as a result of intron 4 retention and encoding a C-terminal peptide sequence of twenty-one amino acid residues. HLA-G6 comprises $\alpha 1$ and $\alpha 3$ domains plus a Cterminal peptide sequence of twenty-one amino acid residues encoded by intron 4 retention and lacks transmembrane and cytoplasmic domains. The HLA-G7 isoform has only the $\alpha 1$ domain and lacks transmembrane and cytoplasmic domains as a result of intron 2 retention and encoding a $\mathrm{C}$-terminal peptide sequence of two amino acid residues. All of these isoforms comprise $\alpha 1$ domain [36].

HLA-G expression has been widely studied in various disorders; nevertheless, the HLA-G gene polymorphism 


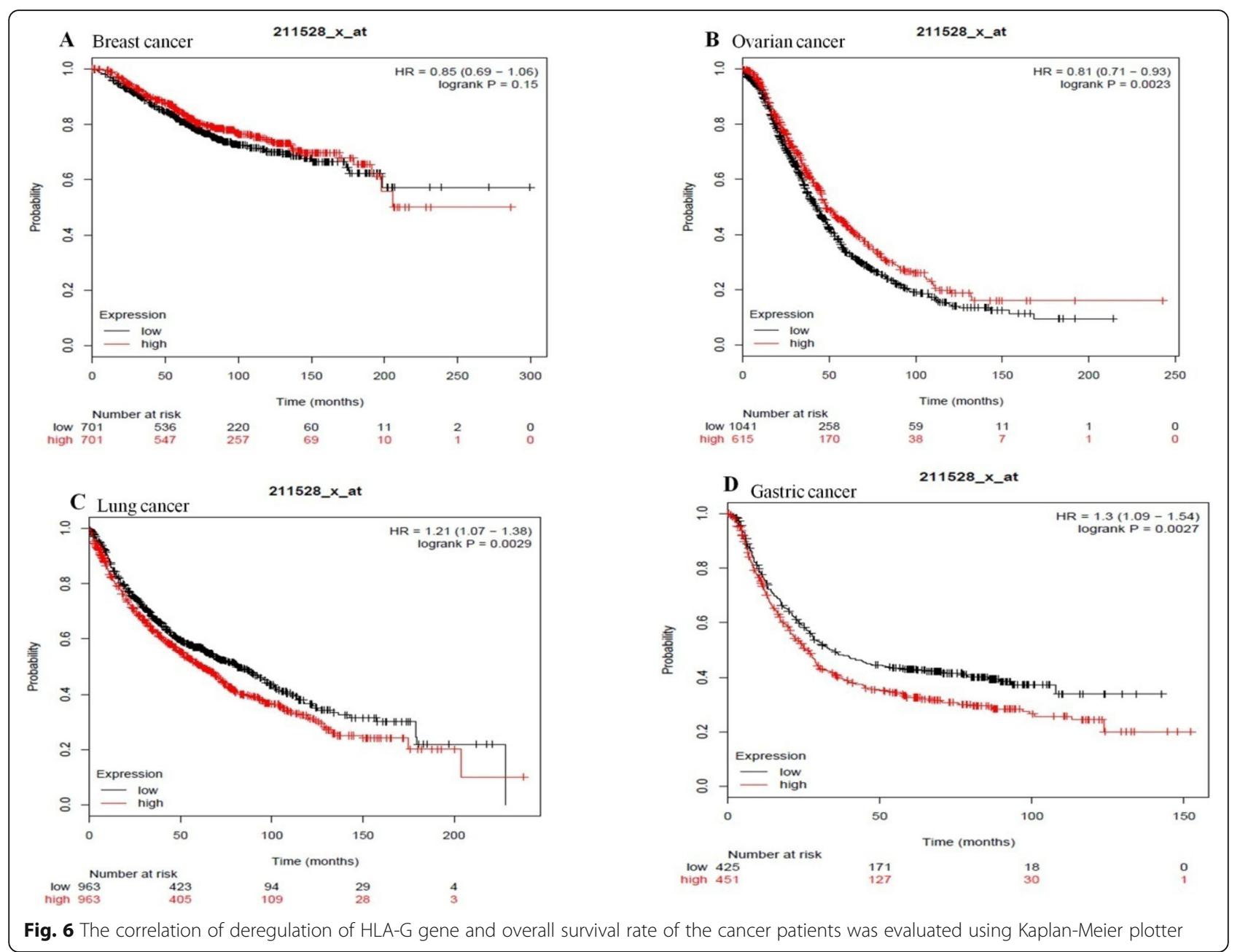

has not been evaluated to the same extent [10]. On the other hand, nearly half of the known gene-related damages for human hereditary diseases are amino acid substitutions. Consequently, screening of polymorphisms using in silico analyses to identify missense SNPs that affect the function of the protein and that are associated with the disease is an important task [29]. Therefore, in the present study, an attempt was made to predict the functional missense SNPs in human HLA-G isoforms. 301 missense SNPs of the human HLA-G gene were retrieved from dbSNP and were submitted to in silico tools to predict the functionally important missense SNPs in HLA-G1 and HLA-G5 and the common most deleterious missense SNPs in membrane-bound isoforms and in the soluble isoforms.

Existing in silico methods have diverse strengths and weaknesses in predicting the effect of nsSNP because every algorithm uses different parameters for prediction $[37,38]$. Therefore, algorithms individually could not be considered as an accurate method for the prediction of functional SNPs [39]. In consequence, screening and prioritizing the candidate functional nsSNPs requires the implementation of different algorithms with different parameters and aspects (e.g. based on evolutionary information and protein structure and/or functional parameters) to combine the advantages of different methods, to enhance the accuracy and reliability of the predictions and to minimize the errors [5, 39-41]. As a general rule, in each study, at least four or five of these tools should be run to obtain a consensus on the effect of single nucleotide polymorphism on the structure and function of the desired protein [37]. In the current investigation, 8 different prediction algorithms were used as follows: SIFT, PROVEAN, PolyPhen-2, I-Mutant 3.0, SNPs\&GO, PhD-SNP, SNAP2 and MUpro for the prediction of deleterious missense SNPs present in HLA-G isoforms. SIFT, PROVEAN, PhD SNP and SNP\&GO tools predict damaging SNPs based only on the sequence of a protein. PolyPhen-2 and SNAP2 tools predict the functional effects of mutations based on the combination 
of protein 3D structure and multiple homolog sequence alignment [37]. I-Mutant 3.0 and MUPro tools investigate the effect of candidate SNPs on protein stability [41]. In our analyses, 35 missense substitutions of all the SNPs in HLA-G 1 isoform were predicted to be most deleterious SNPs by all the programs used. These 35 missense substitutions were classified according to the domain where they were located. Nine (25.71\%) substitutions (rs555347515, rs572025435, rs1475659109, rs1390270595, rs763201540, rs1414848134, rs1260 086927, rs770412396, rs1161818149) are located in the $\alpha 1$ domain, 11 (31.42\%) substitutions (rs17851921, rs5 65858069, rs749006959, rs772834879, rs1317292772, rs556645753, rs867319917, rs748013931, rs780697086, rs1397132797, rs1379742188) are detected in the $\alpha 2$ domain and 15 (42.85\%) substitutions (rs144577485, rs1 472538844, rs770027530, rs1200732770, rs1430565057, rs142596947, rs750238738, rs754527717, rs781774818, rs760500349, rs756652306, rs145097667, rs111233577, rs1265409678, rs765275727) are located in the $\alpha 3$ domain. Thirty-five missense SNPs were found to be the most deleterious on the stability and function of HLA-G 5 isoform. Twelve (34.28\%) substitutions (rs555347515, rs572025435, rs540632198, rs1475659109, rs1390270595, rs763201540, rs1414848134, rs138289952, rs1260086927, rs770412396, rs1161818149, rs776393668) are located in the $\alpha 1$ domain, 12 (34.28\%) substitutions (rs17851921, rs565858069, rs749006959, rs772834879, rs1317292772, rs556645753, rs867319917, rs748013931, rs780697086, rs1397132797, rs1438362414, rs1379742188) are sited in the $\alpha 2$ domain and $11 \quad(31.42 \%)$ substitutions (rs144577485, rs1472538844, rs770027530, rs120073 2770, rs1430565057, rs142596947, rs750238738, rs78 1774818, rs760500349, rs145097667, rs765275727) are located in the $\alpha 3$ domain. Eight missense mutations in the $\alpha 1$ domain with positions M29K, R30S, Y51C, D53N/Y, D54V, Q96P, L102P and L105Q/P among all membrane-bound HLA-G isoforms and with positions M29K, F32C, Y51C, D53N/Y, D54V, Q96P, L102P, L105P between all soluble HLA-G isoforms were predicted as common deleterious missense mutations.

Evidence indicates that all three domains of the heavy chain of HLA-G molecule are involved in inhibiting immune response through interactions with other molecules, for instance, the $\alpha 1$ domain is an important KIR2DL4 recognition site and the LILRB1, LILRB2 and CD8 molecules interact with the $\alpha 3$ domain. Nucleotide variations in these domains may affect the function of the HLA-G molecule. For example, the mutations around domain $\alpha 1$ and $\alpha 2$ affect peptide loading, peptide diversity, and T-cell recognition [10, 15, 42].

In this study, the selected variations were further investigated by other servers. For the rational prioritization of the selected most deleterious SNPs for further studies, an analysis of the evolutionary conservation of selected missense mutations was performed by ConSurf. The amino acids at the conserved regions of protein across species are biologically and functionally very important and SNPs that alter these amino acids may lead to structural and functional changes in the protein $[29,31]$. We have shown that the selected deleterious SNPs in HLA-G1, HLA-G5, the membrane isoforms and the soluble isoforms were mostly in conserved positions and were functional and structural residues, which indicate these SNPs can be deleterious. The MutPred2 web-server predicted the possible molecular mechanisms that result from selected deleterious missense SNPs. The majority of the selected deleterious SNPs were predicted as 'pathogenic' (a g score greater than 0.5) and they are depicted as actionable, confident, and very confident hypotheses based on the $\mathrm{g}$ score and $p$ score. The most predicted effects of very confident hypotheses in HLA-G1 and HLA-G5 were altered transmembrane protein and altered ordered interface. There was not any common predicted effect as very confident hypothesis among all of the membrane-bound HLA-G isoforms. The common predicted effect as very confident hypothesis among all of the soluble HLA-G isoforms was altered ordered interface resulting from $\mathrm{F} 32 \mathrm{C}$ substitution. HOPE investigated the structural effects of the selected deleterious missense SNPs in HLA-G isoforms. The results revealed that nsSNPs are located in each of the three domains $(\alpha 1, \alpha 2$ and $\alpha 3$ ) of HLA-G. Since the function of any protein depends straightly on its tertiary structure, the modification in the structure of the domain can disrupt its function. The native protein $3 \mathrm{D}$ structures are very necessary for better understanding of the functional and structural effect of mutations. In the present study, because the 3D structure of all HLA-G isoforms is not available yet in the PDB database [43]; 3D structural models of native HLA-G isoforms were constructed by I-Tasser server and were visualized using Chimera software. Further, Chimera software was used to visualize the structural consequences of amino acid changes.

The HLA-G promoter region is special in the class of the HLA genes. The 5' UTR and 3' UTR regions of HLA-G gene display many polymorphic sites that may affect HLA-G expression and therefore tissue distribution in healthy and pathological conditions [10]. UTRscan analyzed the $5^{\prime}$ and $3^{\prime}$ UTR SNPs of the HLA-G gene. Two SNPs in the $5^{\prime}$ UTR were determined to create the functional patterns. The rs182801644 was related to creation of the functional pattern of uORF and rs771111444 was related to creation of the functional patterns of UORF and IRES in 5'UTR. The creation of uORF due to SNPs can deregulate the downstream original ORF expression and therefore be the cause of pathological conditions [44]. Furthermore, the presence of new IRES due to SNPs affects the regulation of mRNA translation [45]. To better understand the 
consequences of these UTR SNPs, investigation at the functional levels is needed.

PolymiRTS predicted that 5 functional SNPs are present in the HLA-G mRNA 3' UTR, two of which them disrupt 9 target sites of the miRNA and all five SNPs create 15 new miRNA target sites. MicroRNAs play an important role in translation regulation. Thus disrupting or creating the microRNA target sites influences the regulation of gene and may lead to pathological conditions [10].

STRING analysis is a global way to understand protein-protein interactions. Any change in protein structure and function can affect its ability to interact with other molecules. STRING map showed the interaction of HLA-G with 10 different proteins. Some experimental studies confirm the interaction of HLA-G with these predicted proteins $[9,10,14,15,17,18$, 46-53].

Lastly, the outcomes obtained from Kaplan Meier bioinformatics analyses indicated that the HLA-G gene deregulation affected the overall survival rate of patients with ovarian, lung and gastric cancer and had the prognostic significance. However, there are some controversies in relation to published original studies as presented in Table 7.

Altogether, the findings of the analyses displayed probable alterations that may disrupt the structure and function of HLA-G protein. The deleterious missense mutations determined in this inspection may have functional effects in HLA-G deregulation and may lead to pathological conditions like cancer.

\section{Conclusion}

The implementation of in silico SNP prioritization methods suggests a remarkable framework for the recognition of functional SNPs by reducing the number of alterations that should be screened in molecular studies. Further validation of the results obtained from the current study is recommended using clinical and/or laboratory investigations.

\section{Methods}

Extracting SNPs and protein sequences of HLA-G isoforms from the databases

In December 2018, NCBI dbSNP database [74] (https:// www.ncbi.nlm.nih.gov/snp/) was used to collect information of missense nsSNPs and SNPs in the UTRs of human HLA-G gene. The amino acid sequences of seven human HLA-G isoforms (UniProt ID: P17693-1, P17693-2, P17693-3, P17693-4, P17693-5, P17693-6 and P17693-7) were obtained from the UniProt database [75] (https://www.uniprot.org/uniprot/P17693) in FASTA format for the next stages in this study.
Predicting the most deleterious missense nsSNPs We used eight online bioinformatics tools (SIFT, PROVEAN, PolyPhen-2, I-Mutant 3.0, SNPs\&GO, PhD-SNP, SNAP2 and MUpro) to increase the precision of prediction of the most deleterious missense nsSNPs. Missense nsSNPs found to be most deleterious using these eight tools were further analyzed by several other programs in the next stages.

Sorting intolerant from tolerant (SIFT) [76] (available at https://sift.bii.a-star.edu.sg/) tool expresses whether a missense mutation at special position effects on the structure and function of protein molecule based on sequence homology and the physiochemical characteristics of substituted amino acid. SIFT computes the normalized probability score (SIFT score) for each substitution. The SIFT score has a range of 0.0 to 1.0. The amino acid substitution with a score greater than or equal to 0.05 $(\geq 0.05)$ is predicted as tolerated (polymorphism) whereas a score less than $0.05(<0.05)$ is predicted to be damaging (related to disease).

Protein Variation Effect Analyzer (PROVEAN) (available at provean.jcvi.org/) is another sequence homologybased predictor. It is used to assess the possible functional influence of nonsynonymous (single or multiple nonsynonymous) and in-frame indel (insertions and deletions) variations on a protein. It predicts the variation as deleterious or natural, if the functional impact score is less than or equal to $-2.5(\leq-2.5)$ it is estimated deleterious; score above $-2.5(>-2.5)$ is estimated neutral [77].

Polymorphism Phenotyping version2 (PolyPhen-2) (available at genetics.bwh.harvard.edu/pph2/) is a combination of protein 3D structure and multiple homolog sequence alignment-based method. It predicts the potential consequences of single amino acid substitution on both protein function and structure. The prediction is provided as benign, possibly damaging and probably damaging according to the position-specific independent count (PSIC) scores difference between 2 variants (wild amino acid (aa1) and mutant amino acid (aa2)). PSIC score has a range of 0.0 to 1.0. The amino acid substitution with a score of 0.0 to 0.49 is predicted as benign, with a score of 0.5 to 0.89 is predicted as damaging and with a score of 0.9 to 1 is predicted as probably damaging $[78,79]$.

I-Mutant 3.0 (available at gpcr2.biocomp.unibo.it/cgi/ predictors/I-Mutant3.0/I-Mutant3.0.cgi) is a web server including Support Vector Machine (SVM) based predictors suite. It predicts the effect of a particular amino acid substitution on the stability of protein under default parameters (at room temperature and neutral $\mathrm{pH}$ ) starting from the protein sequence, mutational position and the corresponding novel residue. The protein stability change can disturb both protein function and structure 
Table 7 The correlation of deregulation of HLA-G gene and overall survival rate of the cancer patients as reported by previous studies in comparison with our results

\begin{tabular}{lll}
\hline $\begin{array}{l}\text { Type of } \\
\text { cancer }\end{array}$ & Results found in our study & Results found in some previous studies \\
\hline Breast & $\mathrm{HLA}-\mathrm{G}$ gene in breast cancer had a hazard ratio $(\mathrm{HR})=$ & In the whole cohort of patients, HLA-G showed no \\
Cancer & $0.85(95 \% \mathrm{Cl}, 0.69-1.06)$ and log-rank $p$-value $=0.15 ;$ & $\begin{array}{l}\text { statistically significant difference in outcome between } \\
\text { [54] }\end{array}$
\end{tabular}

$$
(P=0.74) \text {. }
$$

Breast cancer patients with positive HLA-G expression had a lower survival rate in comparison with negative

HLA-G expression patients $(P=0.028)$.

HLA-G upregulated expression was confirmed in breast Yes cancer. HLA-G was associated with both improved relapse-free survival and overall survival.

The expression of HLA-G was significantly higher in Yes invasive ductal breast cancer patients with shorter survival time $(P=0.03)$.

Breast cancer patients with HLA-G-positive tumor cells No had shorter disease-free survival, though not significantly $(P=0.14)$.

Ovarian The relation between the high expression of HLA-G

Cancer gene and more survival rate was statistically significant (less number of patients at risk) $(\mathrm{HR}=0.81(95 \% \mathrm{Cl}, 0.71$ -0.93 ) and log-rank $p$-value $=0.0023$ )
Lung $\quad H L A-G$ gene in lung cancer had a $\mathrm{HR}=1.21(95 \% \mathrm{Cl}$, cancer result was statistically significant (the relation between the low expression of HLA-G gene and more survival rate)
Ovarian cancer patients with HLA-G expression $>17 \%$ Yes showed poor survival than those with HLA-G expression $<17 \%$ group with a $P$ value of 0.04 .

The HLA-G5/-G6 was expressed in 79.7\% (94/118) of ovarian cancer lesions. lesion HLA-G5/-G6 expression was unrelated to clinicoparameters including histological type, patient age, FIGO stages and patient survival.

Survival was prolonged when ovarian tumors expressed No HLA-G $(P=0.008)$ and HLA-G was an independent predictor for better survival $(P=0.011)$. Furthermore, longer progression-free survival $(P=0.036)$ and response to chemotherapy $(P=0.014)$ was correlated with expression of HLA-G.

The Kaplan-Meier analysis demonstrated no significant Yes association between survival and HLA-G expression status in ovarian carcinoma patients.

The Higher sHLA-G level above the median $(\geq 50 \mathrm{U} / \mathrm{ml}) \quad$ No in patients is associated with statistically significant shorter survival time in comparison to the lower sHLA-G expression $(P<0.0001)$.

sHLA-G expression was observed in 34.0\% (45/131) of Yes the NSCLC lesions, which was unrelated to patient survival.

Plasma sHLA-G above the median level ( $\geq$ median, 32.0 $\mathrm{U} / \mathrm{ml}$ ) in NSCLC patients is strongly associated with shorter survival time $(P=0.044)$.

Patients with sHLA-G $<40 \mathrm{ng} / \mathrm{ml}(p=0.073)$ showed No prolonged overall survival.

Patients with HLA-G positive tumors had a significantly shorter survival time than those with tumors that were HLA-G negative $(P=0.001)$.

Survival analyses were shown that the HLA class I loss was correlated to recurrence-free survival time.

Gastric carcinoma patients with HLA-G positive tumors had a significantly shorter survival time than those patients with tumors that were HLA-G negative ( $P=$ .001). 
Table 7 The correlation of deregulation of HLA-G gene and overall survival rate of the cancer patients as reported by previous studies in comparison with our results (Continued)

\begin{tabular}{|c|c|c|c|c|}
\hline $\begin{array}{l}\text { Type of } \\
\text { cancer }\end{array}$ & Results found in our study & Results found in some previous studies & Controversy & Reference \\
\hline \multirow[t]{4}{*}{$\begin{array}{l}\text { Gastric } \\
\text { cancer }\end{array}$} & \multirow{4}{*}{$\begin{array}{l}\text { HLA-G gene in gastric cancer had a } \mathrm{HR}=1.3(95 \% \mathrm{Cl} \text {, } \\
1.09-1.54) \text { and log-rank } p \text {-value }=0.0027 \text {; therefore the } \\
\text { result was statistically significant (the relation between } \\
\text { the low expression of HLA-G gene and more survival } \\
\text { rate. }\end{array}$} & $\begin{array}{l}\text { Kaplan-Meier analyses indicated that patients with HLA- } \\
\text { G-positive gastric cancer had a poorer prognosis than } \\
\text { those with HLA-G negative gastric cancer }(P=0.008) \text {. }\end{array}$ & No & [70] \\
\hline & & $\begin{array}{l}\text { The overall median survival was worse in gastric } \\
\text { adenocarcinoma patients with HLA-G-positive tumors } \\
\text { compared to those with HLA-G-negative tumors } \\
(p<0.0001) \text {. }\end{array}$ & No & [71] \\
\hline & & $\begin{array}{l}\text { Kaplan-Meier analysis showed that gastric cancer } \\
\text { patients with HLA-G expression had a significantly } \\
\text { poorer overall survival than those without HLA-G } \\
\text { expression at } 5 \text { years after the operation. }\end{array}$ & No & [72] \\
\hline & & $\begin{array}{l}\text { The 5-year survival rate of gastric cancer patients in the } \\
\text { HLA-G-positive group was significantly higher than the } \\
\text { HLA-G-negative group. }\end{array}$ & Yes & [73] \\
\hline
\end{tabular}

[80]. I-Mutant 3.0 predicts the protein stability change in the unit of change in Gibbs free energy $(\Delta \Delta G$ or DDG). The DDG value $(\mathrm{kcal} / \mathrm{mol})$ is computed from the unfolding Gibbs free energy value of the mutant protein minus the unfolding Gibbs free energy value of native protein. The prediction is classified into three categories: neutral stability of the mutated protein $(-0.5 \leq \mathrm{DDG} \leq$ $0.5 \mathrm{kcal} / \mathrm{mol})$, a large decrease of stability of the mutated protein $(\leq-0.5 \mathrm{kcal} / \mathrm{mol})$ and large increase of stability of the mutated protein $(>0.5 \mathrm{kcal} / \mathrm{mol})[81]$.

Single Nucleotide Polymorphism Database (SNPs) \& Gene Ontology (GO) (SNPs\&GO) (available at snps.biofold.org/ snps-and-go/snps-and-go.html) is a GO-integrated and single SVM-based predictor. It predicts whether an amino acid substitution is disease-associated or not using functional GO terms, 3D protein structure and protein sequence evolutionary information. The amino acid substitution is associated with the disease if the probability score is greater than $0.5(>0.5)$ [82].

Predictor of human Deleterious Single Nucleotide Polymorphisms (PhD-SNP) (available at snps.biofold. org/phd-snp/phd-snp.html) is a support vector machine (SVM) based server. This server determines whether a certain amino acid substitution is related to disease or neutral by protein sequence information, protein structure, conservation and solvent accessibility. The output is a probability index with a score of 0.0 to 1.0 , when the score is higher than 0.5 , the substituted amino acid is pathogenic [77, 81].

Screening for Non-Acceptable Polymorphisms (SNAP2) (available at https://rostlab.org/services/snap/) is a neural network-based tool that classifies amino acid substitutions into effective and neutral on protein function by taking a diversity of sequences and different characteristics into consideration. SNAP2 provides a list of all possible substitutions within the protein sequence with a score, functional effect (neutral or effect) and expected accuracy for any replacement. The expected accuracy shows the level of confidence for each prediction. The results are also displayed in heat map representation [83-85].

MUpro (available at mupro.proteomics.ics.uci.edu/) uses the Support Vector Machine (SVM) to assess the variation in the stability of the protein consequent to amino acid substitutions. The output is a confidence score among -1 and 1 . A confidence score $<0$ indicates the substituted amino acid decreases the stability and a score $>0$ indicates the substituted amino acid increases the stability [86].

\section{Selecting the most deleterious missense nsSNPs for further study}

Missense nsSNPs that were predicted deleterious by all eight servers were selected for further study. The precision of prediction increases to a greater extent by incorporating the scores of all eight servers.

\section{Predicting the evolutionary conservation of the most deleterious missense nsSNPs by ConSurf}

ConSurf web-server (available at consurf.tau.ac.il/) estimates the evolutionary conservation of each residue in a protein utilizing a Bayesian algorithm which often provides the possibility of identifying key structural and functional residues. The extent of conservation of residue at a specific position in a protein was computed by phylogenetic information of close homologous sequences. The measure of residue conservation is shown by the conservation score along with the color scheme as follows: $1-4$ variable, 5-6 average, and 7-9 conserved. The ConSurf web - server also determines the buried (b) or exposed (e) residues of protein according to the HHPred 3D model. A residue is predicted functional residue if it is very conserved and exposed and a structural residue is predicted if it is very conserved and buried $[87,88]$. 


\section{Studying the most deleterious missense nsSNPs by MutPred2 server}

MutPred is a bioinformatics web server (available at mutpred.mutdb.org/). It predicts whether a particular missense mutation in a human protein is diseaseassociated or not, along with its structural and functional effects (effective molecular characteristics). The result of MutPred consists of two important scores (general (g) score and top 5 molecular properties score (p)), affected PROSITE and ELM motifs and changes of different structural and functional properties. The g score (MutPred score) expresses the probability that the missense mutation is disease-related. The g score is between 0.0 and 1.0. The g score $>0.5$ means the substituted amino acid is probably pathogenic and if $g$ score is > 0.75 , the mutation is more assurance pathogenic. The top 5 molecular properties score (p) is a $P$-value that indicates whether predicted changes of functional and structural characteristics of the protein due to the particular missense mutation are statistically significant. The predicted change is confident if $p$-value is less than $0.05(<0.05)$ and is very confident if $\mathrm{p}$-value is less than $0.01(<0.01)$. The given coalescences of high levels of $g$ scores and low levels of $\mathrm{p}$ scores are called hypotheses. Any prediction according to the scores is put in one of these 3 groups: very confident hypotheses $(\mathrm{g}>0.75$ and $p<0.01$ ), confident hypotheses $(\mathrm{g}>0.75$ and $p<0.05$ ) and actionable hypotheses $(\mathrm{g}>0.5$ and $\mathrm{p}<0.05[89,90]$.

\section{Analyzing the effects of the most deleterious missense SNPs on the 3D structure of the HLA-G isoforms by HOPE project}

Project Have yOur Protein Explained (HOPE) is a web server (available at www.cmbi.ru.nl/hope/) that was used for the investigation of the impacts of a missense mutation on the native protein structure. HOPE will roll up and incorporate available information from UniProtKB, protein's 3D structure and DAS-servers. As regards the exact 3D-structures of some HLA-G protein isoforms are unknown; HOPE built the model of them based on homologous structures. HOPE processes the gathered data and produces a report, including schematic structures of the wild-type and the mutant amino acids, differences in the properties of wild-type and mutant amino acids and the impacts of a substituted amino acid on the protein structure along with figures and animations [91].

\section{Simulating the three-dimensional (3D) structure of HLA-G isoforms by I-TASSER}

To investigate the impact of missense mutations on the structure protein, simulating the protein structure is essential. Iterative Threading ASSEmbly Refinement (ITASSER) (available at https://zhanglab.ccmb.med.umich.
edu/I-TASSER/) is a united program to create the complete protein model and predict protein function based upon the sequence-to-structure-to-function paradigm. Therefore, we used I-TASSER to achieve the highquality three-dimensional (3D) models of HLA-G protein isoforms by submitting their amino acid sequences in FASTA format. The models are created by excising continuous fragments from threading alignments and iterative structural assembly simulations and their functions are derived by matching the 3D models with other known proteins structurally. I-TASSER produces a report, including predicted secondary and tertiary structures, functional annotations and Gene Ontology terms. The accuracy of predicted models is reflected in the form of the confidence score (C-score). The C-score range is between -5 and 2 . The more values of $\mathrm{C}$-score display higher confidence for the predicted model. Five three-dimensional (3D) models were created for each HLA-G protein isoform and the best model was selected according to C-score values [92, 93].

\section{Analyzing changes in HLA-G isoforms 3D structure due to amino acid substitution by UCSF chimera}

UCSF Chimera is a program for molecular visualization, molecular structures study and related data (available at https://www.cgl.ucsf.edu/chimera/). The structures of the HLA-G isoforms predicted with I-TASSER in PDB formatted structure files were visualized by Chimera. Chimera was also used to achieve the $3 \mathrm{D}$ mutated models of the wild models of HLA-G isoforms with the most deleterious missense SNPs predicted in this project. The outputs are graphical models [94].

\section{Founding functional SNPs in UTR by the UTRscan (available at http://itbtools.ba.itb.cnr.it/utrscan)}

This tool is for scrutinizing UTR functional elements throughout user-submitted sequence data for any of the patterns collected in the UTRsite and UTR databases. UTRsite is a pile of functional sequence patterns found in $5^{\prime}$ and $3^{\prime}$ UTR sequences. If two or three sequences of each particular UTR SNP are concluded to have various functional patterns, specific UTR SNP is determined to have functional significance [95].

\section{PolymiRTS database 3.0 (polymorphism in microRNAs and their target sites) (available at compbio.uthsc.edu/ miRSNP/)}

PolymiRTS is a database to analyze the 3'UTR regions of mRNAs in Homo sapiens and mouse for SNPs and INDELs variations in microRNA target sites. The polymorphisms of microRNA target sites may alter miRNAmRNA interactions and accordingly gene expressions. The variations are divided into four categories according to their effect: "D" (the derived allele disrupts a 
conserved miRNA site), "N" (the derived allele disrupts a nonconserved miRNA site), "C" (the derived allele creates a new miRNA site) and "O" (the ancestral allele cannot be determined). " $\mathrm{D}$ " and "C" groups are most likely to have functional effects because they may lead to loss of normal repression and abnormal gene repression control, respectively. We submitted the HLA-G gene symbol to the program and the analysis was performed automatically on the transcript variant 2 (transcript ID: NM_002127) and functional SNPs were determined [96].

\section{Predicting protein-protein interactions by search tool for the retrieval of interacting proteins (STRING) (available at http://string-db.org/)}

STRING is a database of protein-protein interactions. The database contains data from empirical evidences, computational prediction tools and collections of universal text. This provided availability to both experimental and theoretical interaction data of HLA-G $[97,98]$.

\section{Kaplan-Meier plotter analysis (KM plotter) (available at https://kmplot.com/analysis/)}

The Kaplan Meier plotter is a tool to evaluate the impact of 54,000 genes on survival in 21 types of cancer using the microarray gene expression data. A meta-analysis based detection and validation of biomarkers for cancer patients is the primary aim of Kaplan-Meier. The 211528_x_at probe was used for HLA-G gene. Here, the overall survival (OS) is the period of time from the start of a change in specific gene expression (decrease or increase expression) for a cancer, that patients diagnosed with it are still alive. The expression in patients for each cancer was graded and allocated high and low expression groups according to the median level. The overall survival analysis was performed on 1402 cases of breast cancer, 1656 cases of ovarian cancer, 1926 cases of lung cancer and 876 cases of gastric cancer. These two groups of patients for cancer listed above were compared and the survival was evaluated. The $p$-values less than 0.05 were regarded as statistically significant [99-102].

\section{Supplementary information}

Supplementary information accompanies this paper at https://doi.org/10. 1186/s12863-020-00890-y.

\section{Additional file 1:}

Additional file 2: Table 5. Analysis of structural effects of deleterious SNPs on HLA-G1 by Project HOPE

Additional file 3: Table 6. Five models predicted for each human HLA$\mathrm{G}$ isoform by L-TASSER

Additional file 4: Table 7. Structural representations of native isoforms of HLA-G predicted with I-TASSE and visualized with UCSF Chimera

Additional file 5: Table 8. Graphical representations of amino acid changes due to the most deleterious SNPs in isoform 1

\section{Abbreviations}

SNPs: Single-Nucleotide Polymorphisms; MHC: Major histocompatibility complex; HLA: Human leukocyte antigen; dNK: Decidual NK;

UTR: Untranslated regions

\section{Acknowledgements}

The authors would like to thank Mojdeh Riahi for her useful comments for using some servers.

\section{Authors' contributions}

M.E.B conceived of the presented idea. All authors have revised it. E.E collected the information about SNPs from databases, analyzed them with different algorithms and identified the deleterious ones and preformed the rest of the analyses. F.A participated in the analysis of some data. All authors contributed to the interpretation of the results. E.E wrote the initial manuscript and designed the figures. F.A wrote some parts of the manuscript. M.E.B and S.M.K edited the manuscript. M.E.B and E.E prepared the manuscript for submission. All authors read and approved the final manuscript.

\section{Funding}

Not applicable.

\section{Availability of data and materials}

SNPS' information used in present study were retrieved from NCBI dbSNP database (https://www.ncbi.nlm.nih.gov/snp/) [74]. The rsID of SNPs and their information (allele change, residue change, global minor allele frequency (MAF), and position of substitution) retrieved from NCBI dbSNP database and their corresponding IMGT/HLA alleles from https://raw.githubusercontent com/ANHIG/IMGTHLA/Latest/ alignments/G_prot.txt) were presented in supplementary data (Table 1). The MAF of SNPs was also obtained from the dbSNP GeneView page (https://www.ncbi.nlm.nih.gov/projects/SNP/snp_ref. cgi? showRare $=$ on\&chooseRs $=$ coding $\& g o=$ Go\&locusld $=3135$ ) and is shown in supplementary Table 1. The amino acid sequences of HLA-G isoforms were achieved from the UniProt database (https://www.uniprot.org/ uniprot/P17693) [75]. The tools used for prediction of the most deleterious missense nsSNPs were SIFT (https://sift.bii.a-star.edu.sg/) [76], PROVEAN (provean.jcvi.org/) [77], PolyPhen-2 (genetics.bwh.harvard.edu/pph2/) [78, 79], IMutant 3.0 (gpcr2.biocomp.unibo.it/cgi/predictors/l-Mutant3.0/l-

Mutant3.0.cgi) [80], SNPs\&GO (snps.biofold.org/snps-and-go/snps-and-go. html) [82], PhD-SNP (snps.biofold.org/phd-snp/phd-snp.html) [77, 81], SNAP2 (https://rostlab.org/services/snap/) [83-85] and MUpro (mupro.proteomics.ics. uci.edu/) [86]. ConSurf web-server (consurf.tau.ac.il/) [87, 88] estimates the evolutionary conservation of the most deleterious missense nsSNPs. The structural and functional effects of predicted SNPs were investigated with the MutPred web server (mutpred.mutdb.org/) [89, 90] and HOPE web server (www.cmbi.ru.nl/hope/) [91]. The 3D models of HLA-G protein isoforms were achieved using I-TASSER (https://zhanglab.ccmb.med.umich.edu/l-TASSER/). Chimera was used for analyzing changes in 3D structures due to amino acid substitution (https://www.cgl.ucsf.edu/chimera/) [94]. Founding functional SNPs in UTR was performed using the UTRscan (http://itbtools.ba.itb.cnr.it/ utrscan) [95]. The used database for analysis of SNPs in microRNA target sites was PolymiRTS (compbio.uthsc.edu/miRSNP/) [96]. Interaction of the HLA-G protein with other proteins was investigated with the STRING database (http://string-db.org/) $[97,98]$. The effect of dysregulation expression of HLA$G$ on survival in four types of cancer was assessed using the Kaplan Meier plotter (https://kmplot.com/analysis/) [99-102].

Ethics approval and consent to participate

Not applicable.

Consent for publication

Not applicable.

\section{Competing interests}

The authors declare that they have no competing interests.

\section{Author details}

${ }^{1}$ Department of Genetics, Faculty of Medicine, Shahid Sadoughi University of Medical Sciences, Yazd, Iran. ${ }^{2}$ Department of Genetics, Faculty of Basic 
Sciences, Shahrekord University, Shahrekord, Iran. ${ }^{3}$ Research Institute of Biotechnology, Shahrekord University, Shahrekord, Iran.

\section{Received: 24 January 2020 Accepted: 19 July 2020 Published online: 31 August 2020}

\section{References}

1. Group ISMW. A map of human genome sequence variation containing 1.42 million single nucleotide polymorphisms. Nature. 2001;409(6822):928.

2. Ding C, Jin S. High-throughput methods for SNP genotyping. In: Single Nucleotide Polymorphisms. Springer, Methods Mol Biol. 2009:578:245-54. https://link.springer.com/protocol/10.1007/978-1-60327-411-1_16.

3. Rajasekaran R, Doss CGP, Sudandiradoss C, Ramanathan K, Sethumadhavan R. In silico analysis of structural and functional consequences in p16INK4A by deleterious nsSNPs associated CDKN2A gene in malignant melanoma. Biochimie. 2008;90(10):1523-9.

4. Dakal TC, Kala D, Dhiman G, Yadav V, Krokhotin A, Dokholyan NV. Predicting the functional consequences of non-synonymous single nucleotide polymorphisms in IL8 gene. Sci Rep. 2017;7(1):6525.

5. Bhatnager R, Dang AS. Comprehensive in-silico prediction of damage associated SNPs in human Prolidase gene. Sci Rep. 2018;8(1):9430.

6. Janeway Jr CA, Travers P, Walport M, Shlomchik MJ. The major histocompatibility complex and its functions. In: Immunobiology: The Immune System in Health and Disease 5th edition. New York: Garland Science; 2001. Available from: http://www.ncbi.nlm.nih.gov/books/NBK2 7156/. ISBN-10: 0-8153-3642-X.

7. Mosaad Y. Clinical role of human leukocyte antigen in health and disease. Scand J Immunol. 2015;82(4):283-306.

8. Hassan M, Dowd A, Ibrahim F, Mohamed A, Kaheel H, Hassan M. In silico analysis of single nucleotide polymorphisms (SNPs) in human HLA-A and HLA-B genes responsible for renal transplantation rejection. Eur Acad Res. 2014;2(3):3627-46.

9. HoWang Yin K-Y, Loustau M, Wu J, Alegre E, Daouya M, Caumartin J, Sousa S, Horuzsko A, Carosella ED, LeMaoult J. Multimeric structures of HLA-G isoforms function through differential binding to LILRB receptors. Cell Mol Life Sci. 2012;69(23):4041-9.

10. Donadi EA, Castelli EC, Arnaiz-Villena A, Roger M, Rey D, Moreau P. Implications of the polymorphism of HLA-G on its function, regulation, evolution and disease association. Cell Mol Life Sci. 2011;68(3):369-95.

11. Schwich E, Rebmann V, Michita RT, Rohn H, Voncken JW, Horn PA, Kimmig R, Kasimir-Bauer S, Buderath P. HLA-G 3' untranslated region variants+ $3187 \mathrm{G} / \mathrm{G},+3196 \mathrm{G} / \mathrm{G}$ and $+3035 \mathrm{~T}$ define diametrical clinical status and disease outcome in epithelial ovarian cancer. Sci Rep. 2019;9(1):5407.

12. Tronik-Le Roux D, Renard J, Vérine J, Renault V, Tubacher E, LeMaoult J, Rouas-Freiss N, Deleuze JF, Desgrandschamps F, Carosella ED. Novel landscape of HLA-G isoforms expressed in clear cell renal cell carcinoma patients. Mol Oncol. 2017;11(11):1561-78.

13. Rizzo R, Trentini A, Bortolotti D, Manfrinato MC, Rotola A, Castellazzi M, Melchiorri L, Di Luca D, Dallocchio F, Fainardi E. Matrix metalloproteinase-2 (MMP-2) generates soluble HLA-G1 by cell surface proteolytic shedding. Mol Cell Biochem. 2013;381(1-2):243-55.

14. Bainbridge $D$, Ellis $S$, Le Bouteiller P, Sargent I. HLA-G remains a mystery. Trends Immunol. 2001;22(10):548-52.

15. Yie S-M. HLA-G (major histocompatibility complex, class I, G). Atlas Genetics Cytogenetics Oncol Haematol. 2012;16(6):403-11.

16. Menier C, Rouas-Freiss N, Carosella ED. The HLA-G non classical MHC class I molecule is expressed in cancer with poor prognosis. Implications in tumour escape from immune system and clinical applications. Atlas Genetics Cytogenetics Oncol Haematol. 2009;13(7):531-42.

17. Ho G-GT, Heinen F, Stieglitz F, Blasczyk R, Bade-Doeding C. Dynamic interaction between immune escape mechanism and HLA-lb regulation. In: Immunogenetics. Rezaei N, Ed. London: IntechOpen Limited; 2018. p. 17982. https://www.intechopen.com/books/immunogenetics/dynamicinteraction-between-immune-escape-mechanism-and-hla-ib-regulation.

18. Alegre E, Rizzo R, Bortolotti D, Fernandez-Landázuri S, Fainardi E, González A. Some basic aspects of HLA-G biology. J Immunol Res. 2014;2014:657625, 10 pages. https://doi.org/10.1155/2014/657625.

19. Gregori S. Hla-G-mediated immune tolerance: past and new outlooks. Front Immunol. 2016;7:653.
20. Lin A, Yan W-H. Heterogeneity of HLA-G expression in cancers: facing the challenges. Front Immunol. 2018;9:2164. https:/www.frontiersin.org/article/1 0.3389/fimmu.2018.02164.

21. Moreau P, Flajollet $\mathrm{S}$, Carosella ED. Non-classical transcriptional regulation of HLA-G: an update. J Cell Mol Med. 2009;13(9b):2973-89.

22. Kamaraj B, Purohit R. In silico screening and molecular dynamics simulation of disease-associated nsSNP in TYRP1 gene and its structural consequences in OCA3. Biomed Res Int. 2013;2013:1-13. https://doi.org/10.1155/2013/697051.

23. Bhagwat M. Searching NCBI's dbSNP database. Curr Protocols Bioinformatics. 2010;32(1):1-9 11-11.19. 18.

24. Ramensky V, Bork P, Sunyaev S. Human non-synonymous SNPs: server and survey. Nucleic Acids Res. 2002;30(17):3894-900.

25. Miller MP, Kumar S. Understanding human disease mutations through the use of interspecific genetic variation. Hum Mol Genet. 2001;10(21):2319-28.

26. Doss CGP, Rajith B, Garwasis N, Mathew PR, Raju AS, Apoorva K, William D, Sadhana N, Himani T, Dike I. Screening of mutations affecting protein stability and dynamics of FGFR1 — a simulation analysis. Appl Transl Genomics. 2012;1:37-43.

27. Pickering $B M$, Willis $A E$. The implications of structured $5^{\prime}$ untranslated regions on translation and disease. In: Seminars in cell \& developmental biology: Elsevier; 2005;16:39-47. https:/doi.org/10.1016/j.semcdb.2004.11.006.

28. Meijer HA, Thomas AA. Control of eukaryotic protein synthesis by upstream open reading frames in the 5 -untranslated region of an mRNA. Biochem J. 2002;367(1):1-11.

29. Masoodi TA, Al Shammari SA, Al-Muammar MN, Alhamdan AA. Screening and evaluation of deleterious SNPs in APOE gene of Alzheimer's disease. Neurol Res Int. 2012;2012:480609, 8 pages. https://doi.org/10.1155/2012/480609.

30. Vignal A, Milan D, SanCristobal M, Eggen A. A review on SNP and other types of molecular markers and their use in animal genetics. Genet Sel Evol. 2002;34(3):275.

31. Kaur T, Thakur K, Singh J, Kamboj SS, Kaur M. Identification of functional SNPs in human LGALS3 gene by in silico analyses. Egypt I Med Hum Genetics. 2017;18(4):321-8.

32. Chen X, Sullivan P. Single nucleotide polymorphism genotyping: biochemistry, protocol, cost and throughput. Pharmacogenomics J. 2003; 3(2):77.

33. Akhoundi F, Parvaneh N, Modjtaba E-B. In silico analysis of deleterious single nucleotide polymorphisms in human BUB1 mitotic checkpoint serine/ threonine kinase B gene. Meta gene. 2016;9:142-50.

34. Smigielski EM, Sirotkin K, Ward M, Sherry ST. dbSNP: a database of single nucleotide polymorphisms. Nucleic Acids Res. 2000;28(1):352-5.

35. Carosella ED, Moreau P, Le Maoult J, Le Discorde M, Dausset J, Rouas-Freiss N. HLA-G molecules: from maternal-fetal tolerance to tissue acceptance. Adv Immunol. 2003:81:199-252.

36. Le Maoult J, Carosella ED. Multimeric polypeptides of HLA-G including alpha1-alpha3 monomers and pharmaceutical uses thereof. In: Google Patents; 2016

37. Hussien A, Osman AA. In Silico Screening and Analysis of SNPs in Human ABCB1 (MDR1) Gene. bioRxiv. 2019:505859. https://www.biorxiv.org/ content/10.1101/505859v1.

38. Mohamoud A, Sheikh H, Hussain M, Ramzan M, El-Harouni AA, Shaik NA, Qasmi ZU, Merican AF, Baig M, Anwar Y. First comprehensive in silico analysis of the functional and structural consequences of SNPs in human GalNAc-T1 gene. Comput Math Methods Med. 2014;2014:904052, 15 pages. https://doi.org/10.1155/2014/904052.

39. Doss CGP, Rajith B. A new insight into structural and functional impact of single-nucleotide polymorphisms in PTEN gene. Cell Biochem Biophys. 2013;66(2):249-63

40. Pires AS, Porto WF, Franco OL, Alencar SA. In silico analyses of deleterious missense SNPs of human apolipoprotein E3. Sci Rep. 2017;7(1):2509.

41. Abdelmoneim AH, Mustafa MI, Mahmoud TA, Murshed NS, Hassan MA. In Silico Analysis and Modeling of Novel Pathogenic Single Nucleotide Polymorphisms (SNPs) in Human CD40LG Gene. bioRxiv. 2019:552596. https://www.biorxiv.org/content/10.1101/552596v1.abstract.

42. Rajagopalan S, Long EO. KIR2DL4 (CD158d): an activation receptor for HLAG. Front Immunol. 2012;3:258.

43. Desai M, Chauhan J. In silico analysis of nsSNPs in human methyl CpG binding protein 2. Meta Gene. 2016;10:1-7.

44. Silva J, Fernandes R, Romão L. Gene expression regulation by upstream open reading frames in rare diseases. J Rare Dis Res Treat. 2017;2(4):33-8. 
45. Ramírez-Bello J, Jiménez-Morales M. Functional implications of single nucleotide polymorphisms (SNPs) in protein-coding and non-coding RNA genes in multifactorial diseases. Gaceta medica de Mexico. 2017;153(2):238-50.

46. Morandi F, Pistoia V. Interactions between HLA-G and HLA-E in physiological and pathological conditions. Front Immunol. 2014;5:394.

47. Shiroishi M, Kuroki K, Rasubala L, Tsumoto K, Kumagai I, Kurimoto E, Kato K, Kohda D, Maenaka K. Structural basis for recognition of the nonclassical MHC molecule HLA-G by the leukocyte lg-like receptor B2 (LILRB2/LIR2/ ILT4/CD85d). Proc Natl Acad Sci. 2006;103(44):16412-7.

48. King A, Hiby S, Verma S, Burrows T, Gardner L, Loke Y. Uterine NK cells and trophoblast HLA class I molecules. Am J Reprod Immunol. 1997;37(6):459-62.

49. Gao GF, Willcox BE, Wyer JR, Boulter JM, O'Callaghan CA, Maenaka K, Stuart DI, Jones EY, Van Der Merwe PA, Bell II. Classical and nonclassical class I major histocompatibility complex molecules exhibit subtle conformational differences that affect binding to CD8aa. J Biol Chem. 2000;275(20):15232-8.

50. Huttlin EL, Bruckner RJ, Paulo JA, Cannon JR, Ting L, Baltier K, Colby G, Gebreab F, Gygi MP, Parzen H. Architecture of the human interactome defines protein communities and disease networks. Nature. 2017:545(7655):505.

51. Huttlin EL, Ting L, Bruckner RJ, Gebreab F, Gygi MP, Szpyt J, Tam S, Zarraga G, Colby G, Baltier K. The BioPlex network: a systematic exploration of the human interactome. Cell. 2015;162(2):425-40.

52. Rajagopalan S, Long EO. A human histocompatibility leukocyte antigen (HLA)-Gspecific receptor expressed on all natural killer cells. J Exp Med. 1999;189(7):1093-100.

53. Shiroishi M, Tsumoto K, Amano K, Shirakihara Y, Colonna M, Braud VM, Allan DS, Makadzange A, Rowland-Jones S, Willcox B. Human inhibitory receptors Iglike transcript 2 (ILT2) and ILT4 compete with CD8 for MHC class I binding and bind preferentially to HLA-G. Proc Natl Acad Sci. 2003;100(15):8856-61.

54. de Kruijf EM, Sajet A, van Nes JG, Natanov R, Putter H, Smit VT, Liefers GJ, van den Elsen PJ, van de Velde CJ, Kuppen PJ. HLA-E and HLA-G expression in classical HLA class I-negative tumors is of prognostic value for clinical outcome of early breast cancer patients. J Immunol. 2010;185(12):7452-9.

55. He X, Dong D-D, Yie S-M, Yang H, Cao M, Ye S-R, Li K, Liu J, Chen J. HLA-G expression in human breast cancer: implications for diagnosis and prognosis, and effect on allocytotoxic lymphocyte response after hormone treatment in vitro. Ann Surg Oncol. 2010;17(5):1459-69.

56. Martínez-Canales S, Cifuentes F, Gregorio MLDR, Serrano-Oviedo L, GalánMoya EM, Amir E, Pandiella A, Győrffy B, Ocaña A. Transcriptomic immunologic signature associated with favorable clinical outcome in basallike breast tumors. PLoS One. 2017;12(5):e0175128.

57. Ramos CS, Gonçalves AS, Marinho LC, Avelino MAG, Saddi VA, Lopes AC, Simões RT, Wastowski IJ. Analysis of HLA-G gene polymorphism and protein expression in invasive breast ductal carcinoma. Hum Immunol. 2014;75(7):667-72.

58. Kleinberg L, Flørenes VA, Skrede M, Dong HP, Nielsen S, McMaster MT, Nesland JM, Shih I-M, Davidson B. Expression of HLA-G in malignant mesothelioma and clinically aggressive breast carcinoma. Virchows Arch. 2006:449(1):31-9.

59. Jung YW, Kim YT, Kim SW, Kim S, Kim JH, Cho NH, Kim JW. Correlation of human leukocyte antigen-G (HLA-G) expression and disease progression in epithelial ovarian cancer. Reprod Sci. 2009;16(11):1103-11.

60. Zhang X, Han Q-Y, Li J-B, Ruan Y-Y, Yan W-H, Lin A. Lesion HLA-G5/-G6 isoforms expression in patients with ovarian cancer. Hum Immunol. 2016; 77(9):780-4

61. Rutten M, Dijk F, Savci-Heijink C, Buist M, Kenter G, van de Vijver M, Jordanova E. HLA-G expression is an independent predictor for improved survival in high grade ovarian carcinomas. J Immunol Res. 2014;2014: 274584. https://doi.org/10.1155/2014/274584.

62. Babay W, Yahia HB, Boujelbene N, Zidi N, Laaribi AB, Kacem D, Ghorbel RB, Boudabous A, Ouzari H-I, Rizzo R. Clinicopathologic significance of HLA-G and HLA-E molecules in Tunisian patients with ovarian carcinoma. Hum Immunol. 2018;79(6):463-70.

63. Amor AB, Beauchemin K, Faucher M-C, Hamzaoui A, Hamzaoui K, Roger M Human leukocyte antigen $\mathrm{G}$ polymorphism and expression are associated with an increased risk of non-small-cell lung cancer and advanced disease stage. PLoS One. 2016;11(8):e0161210.

64. Yan WH, Liu D, Lu HY, Li YY, Zhang X, Lin A. Significance of tumour cell HLA-G5/-G6 isoform expression in discrimination for adenocarcinoma from squamous cell carcinoma in lung cancer patients. J Cell Mol Med. 2015; 19(4):778-85.

65. Lin A, Zhu CC, Chen HX, Chen BF, Zhang X, Zhang JG, Wang Q, Zhou WJ, $\mathrm{Hu}$ W, Yang HH. Clinical relevance and functional implications for human leucocyte antigen-g expression in non-small-cell lung cancer. J Cell Mol Med. 2010;14(9):2318-29.

66. Schütt P, Schütt B, Switala M, Bauer S, Stamatis G, Opalka B, Eberhardt W, Schuler M, Horn PA, Rebmann V. Prognostic relevance of soluble human leukocyte antigen-G and total human leukocyte antigen class I molecules in lung cancer patients. Hum Immunol. 2010;71(5):489-95.

67. S-m Y, Yang H, Ye S-R, Li K, Dong D-D, Lin X-M. Expression of human leucocyte antigen $\mathrm{G}(\mathrm{HLA}-\mathrm{G})$ is associated with prognosis in non-small cell lung cancer. Lung Cancer. 2007;58(2):267-74.

68. Suzuki H, Higuchi M, Hasegawa T, Yonechi A, Ohsugi J, Yamada F, Hoshino M, Shio Y, Fujiu K, Gotoh M. Tissue array analysis of the aberrant expression of HLA class I molecules in human non small cell lung cancer. Gan To Kagaku Ryoho. 2006;33(12):1713-6.

69. S-m Y, Yang H, Ye S-R, Li K, Dong D-D, Lin X-M. Expression of human leukocyte antigen $\mathrm{G}(\mathrm{HLA}-\mathrm{G})$ correlates with poor prognosis in gastric carcinoma. Ann Surg Oncol. 2007;14(10):2721-9.

70. Tuncel T, Karagoz B, Haholu A, Ozgun A, Emirzeoglu L, Bilgi O, Kandemir EG. Immunoregulatory function of HLA-G in gastric cancer. Asian Pac J Cancer Prev. 2013;14(12):7681-4.

71. Murdaca G, Calamaro P, Lantieri F, Pigozzi S, Mastracci L, Grillo F, Magnani O, Ceppa P, Puppo F, Fiocca R. HLA-G expression in gastric carcinoma: clinicopathological correlations and prognostic impact. Virchows Arch. 2018; 473(4):425-33.

72. Du L, Xiao X, Wang C, Zhang X, Zheng N, Wang L, Zhang X, Li W, Wang S, Dong Z. Human leukocyte antigen-G is closely associated with tumor immune escape in gastric cancer by increasing local regulatory $T$ cells. Cancer Sci. 2011;102(7):1272-80.

73. Ishigami S, Natsugoe S, Miyazono F, Nakajo A, Tokuaa K, Matsumoto M, Okumura H, Douchi T, Hokita S, Aikou T. HLA-G expression in gastric cancer. Anticancer Res. 2006;26(3B):2467-72.

74. Sherry ST, Ward M-H, Kholodov M, Baker J, Phan L, Smigielski EM, Sirotkin K. dbSNP: the NCBI database of genetic variation. Nucleic Acids Res. 2001; 29(1):308-11.

75. Consortium U. UniProt: a worldwide hub of protein knowledge. Nucleic Acids Res. 2018;47(D1):D506-15.

76. Kumar P, Henikoff S, Ng PC. Predicting the effects of coding nonsynonymous variants on protein function using the SIFT algorithm. Nat Protoc. 2009:4(7):1073.

77. Hassan MS, Shaalan A, Dessouky M, Abdelnaiem AE, ElHefnawi M. A review study: computational techniques for expecting the impact of non-synonymous single nucleotide variants in human diseases. Gene. 2019;680:20-33.

78. Adzhubei IA, Schmidt S, Peshkin L, Ramensky VE, Gerasimova A, Bork P, Kondrashov AS, Sunyaev SR. A method and server for predicting damaging missense mutations. Nat Methods. 2010;7(4):248.

79. Adzhubei I, Jordan DM, Sunyaev SR. Predicting functional effect of human missense mutations using PolyPhen-2. Curr Protocols Human Genetics. 2013;76(1):7-20 21-27.20. 41.

80. Daggett $V$, Fersht AR. Is there a unifying mechanism for protein folding? Trends Biochem Sci. 2003;28(1):18-25.

81. Capriotti E, Calabrese R, Casadio R. Predicting the insurgence of human genetic diseases associated to single point protein mutations with support vector machines and evolutionary information. Bioinformatics. 2006;22(22): 2729-34.

82. Calabrese R, Capriotti E, Fariselli P, Martelli PL, Casadio R. Functional annotations improve the predictive score of human disease-related mutations in proteins. Hum Mutat. 2009;30(8):1237-44.

83. Bromberg $Y$, Rost B. SNAP: predict effect of non-synonymous polymorphisms on function. Nucleic Acids Res. 2007;35(11):3823-35.

84. Yachdav G, Hecht M, Pasmanik-Chor M, Yeheskel A, Rost B. HeatMapViewer: interactive display of 2D data in biology. F1000Research. 2014;1:3-48. https://www.ncbi.n/m.nih.gov/pmc/articles/PMC4023661/.

85. Hepp D, Gonçalves GL, de Freitas TRO. Prediction of the damage-associated non-synonymous single nucleotide polymorphisms in the human MC1R gene. PLoS One. 2015;10(3):e0121812.

86. Cheng J, Randall A, Baldi P. Prediction of protein stability changes for single-site mutations using support vector machines. Proteins. 2006;62(4): 1125-32.

87. Ashkenazy H, Erez E, Martz E, Pupko T, Ben-Tal N. ConSurf 2010: calculating evolutionary conservation in sequence and structure of proteins and nucleic acids. Nucleic Acids Res. 2010;38(suppl_2):W529-33. 
88. Ashkenazy H, Abadi S, Martz E, Chay O, Mayrose I, Pupko T, Ben-Tal N. ConSurf 2016: an improved methodology to estimate and visualize evolutionary conservation in macromolecules. Nucleic Acids Res. 2016; 44(W1):W344-50.

89. Pejaver V, Urresti J, Lugo-Martinez J, Pagel KA, Lin GN, Nam H-J, Mort M, Cooper DN, Sebat J, lakoucheva LM. MutPred2: inferring the molecular and phenotypic impact of amino acid variants. BioRxiv. 2017;134981. https:// www.biorxiv.org/content/10.1101/134981v1.abstract.

90. Li B, Krishnan VG, Mort ME, Xin F, Kamati KK, Cooper DN, Mooney SD, Radivojac P. Automated inference of molecular mechanisms of disease from amino acid substitutions. Bioinformatics. 2009;25(21):2744-50.

91. Venselaar H, te Beek TA, Kuipers RK, Hekkelman ML, Vriend G. Protein structure analysis of mutations causing inheritable diseases. An e-Science approach with life scientist friendly interfaces. BMC bioinformatics. 2010; 11(1):548.

92. Zhang Y. I-TASSER server for protein 3D structure prediction. BMC bioinformatics. 2008;9(1):40

93. Roy A, Kucukural A, Zhang Y. I-TASSER: a unified platform for automated protein structure and function prediction. Nat Protoc. 2010;5(4):725.

94. Pettersen EF, Goddard TD, Huang CC, Couch GS, Greenblatt DM, Meng EC, Ferrin TE. UCSF chimera-a visualization system for exploratory research and analysis. J Comput Chem. 2004;25(13):1605-12.

95. Grillo G, Turi A, Licciulli F, Mignone F, Liuni S, Banfi S, Gennarino VA, Horner DS, Pavesi G, Picardi E. UTRdb and UTRsite (RELEASE 2010): a collection of sequences and regulatory motifs of the untranslated regions of eukaryotic mRNAs. Nucleic Acids Res. 2009:38(suppl_1):D75-80.

96. Bhattacharya A, Ziebarth JD, Cui Y. PolymiRTS database 3.0: linking polymorphisms in microRNAs and their target sites with human diseases and biological pathways. Nucleic Acids Res. 2013;42(D1):D86-91.

97. Jensen L, Kuhn M, Stark M, Chaffron S, Creevey C, Muller J, Doerks T, Julien P, Roth A, Simonovic M. STRING 8-a global view on proteins and their functional interactions in 630 organisms. Nucleic Acids Res. 2008;37(suppl_ 1):D412-6.

98. Szklarczyk D, Franceschini A, Kuhn M, Simonovic M, Roth A, Minguez P, Doerks T, Stark M, Muller J, Bork P. The STRING database in 2011: functional interaction networks of proteins, globally integrated and scored. Nucleic Acids Res. 2010;39(suppl_1)):D561-8.

99. Györffy B, Lanczky A, Eklund AC, Denkert C, Budczies J, Li Q, Szallasi Z. An online survival analysis tool to rapidly assess the effect of 22,277 genes on breast cancer prognosis using microarray data of 1,809 patients. Breast Cancer Res Treat. 2010;123(3):725-31.

100. Győrffy B, Lánczky A, Szállási Z. Implementing an online tool for genome-wide validation of survival-associated biomarkers in ovarian-cancer using microarray data from 1287 patients. Endocr Relat Cancer. 2012;19(2):197-208.

101. Győrffy B, Surowiak P, Budczies J, Lánczky A. Online survival analysis software to assess the prognostic value of biomarkers using transcriptomic data in non-small-cell lung cancer. PLoS One. 2013;8(12):e82241.

102. Szász AM, Lánczky A, Nagy Á, Förster S, Hark K, Green JE, Boussioutas A, Busuttil R, Szabó A, Győrffy B. Cross-validation of survival associated biomarkers in gastric cancer using transcriptomic data of 1,065 patients. Oncotarget. 2016;7(31):49322.

\section{Publisher's Note}

Springer Nature remains neutral with regard to jurisdictional claims in published maps and institutional affiliations.

\section{Ready to submit your research? Choose BMC and benefit from}

- fast, convenient online submission

- thorough peer review by experienced researchers in your field

- rapid publication on acceptance

- support for research data, including large and complex data types

- gold Open Access which fosters wider collaboration and increased citations

- maximum visibility for your research: over $100 \mathrm{M}$ website views per year

At BMC, research is always in progress.

Learn more biomedcentral.com/submissions 